Aus der Klinik für Klinische Neurophysiologie

(Prof. Dr. W. Paulus)

im Zentrum Neurologische Medizin

der Medizinischen Fakultät der Universität Göttingen

\title{
Kortikale Repräsentation der humanen aurikulären Muskulatur: Eine Untersuchung mittels robotergestützter und neuronavigationsbasierter transkranieller Magnetstimulation
}

$$
\text { INAUGURAL - DISSERTATION }
$$

zur Erlangung des Doktorgrades der Medizinischen Fakultät der Georg-August-Universität zu Göttingen

\author{
vorgelegt von \\ Jonna Meincke \\ aus Göttingen
}

Göttingen 2015 
Dekan: Prof. Dr. rer. nat. H. K. Kroemer

1. Berichterstatter: Prof. Dr. med. D. Liebetanz

2. Berichterstatter: PD Dr. med. M. Rickmann

Tag der mündlichen Prüfung: 13.12.2016 


\section{Inhaltsverzeichnis}

I. Zusammenfassung 5

$\begin{array}{lll}\text { II. } & \text { Abkürzungen } & 7\end{array}$

1. EINLEITUNG

$\begin{array}{lll}1.1 & \text { Die aurikuläre Muskulatur } & 8\end{array}$

$\begin{array}{lll}1.2 & \text { Primär motorischer Kortex } & 10\end{array}$

$\begin{array}{lll}1.3 & \text { Transkranielle Magnetstimulation } & 11\end{array}$

1.4 Motorisch evozierte Potenziale und Elektromyographie 13

1.5 Hotspot und Stimulationsschwelle (threshold) 16

1.6 Mapping von kortikalen motorischen Repräsentationsarealen mittels transkranieller Magnetstimulation 17

$\begin{array}{lll}1.7 & \text { Spulenpositionierung und Neuronavigation } & 18\end{array}$

$\begin{array}{lll}1.8 & \text { Ziele der Untersuchung } & 21\end{array}$

2. MATERIAL UND METHODEN 22

$\begin{array}{lll}2.1 & \text { Versuchsgruppe } & 22\end{array}$

2.2 Versuchsdurchführung 22

2.3 Roboter- und bildgestützte transkranielle Magnetstimulation 25

$\begin{array}{lll}2.4 & \text { Datenanalyse } & 28\end{array}$

3. ERGEBNISSE 32

3.1 Motorisch evozierte Potenziale im Musculus auricularis posterior 32

3.2 Mapping des Musculus auricularis posterior und Musculus interosseus dorsalis I mittels transkranieller Magnetstimulation 
3.3 Kortikale Repräsentation des Musculus auricularis posterior und Musculus interosseus dorsalis I

4. DISKUSSION

4.1 Motorisch evozierte Potenziale im Musculus auricularis posterior im Menschen

4.1.1 Voraktivierung

4.1.2 Latenzen

4.1.3 Blinkreflex

4.1.4 Musculus-auricularis-posterior-Reflex

4.2 Kortikales Repräsentationsareal des Musculus auricularis posterior und Musculus interosseus dorsalis I

4.3 Lokalisation der kortikalen Repräsentation des Musculus auricularis posterior und Musculus interosseus dorsalis I

4.4 Funktionelle Bildgebung während Aktivierung des Musculus auricularis posterior und Musculus interosseus dorsalis I

4.5 Vergleich der Mapping-Ergebnisse der transkraniellen Magnetstimulation mit der funktionellen Magnetresonanztomographie

4.6 Robotergestützte und neuronavigationsbasierte transkranielle Magnetstimulation

5. SCHLUSSFOLGERUNG UND AUSBLICK

6. PERSPEKTIVEN

7. LITERATURVERZEICHNIS 


\section{Zusammenfassung}

Die aurikulären Muskeln sind Teil der durch den N. facialis innervierten Gesichtsmuskulatur, über deren Funktion und Neuroanatomie im Menschen jedoch wenig bekannt ist. In der Literatur werden sie größtenteils als rudimentär beschrieben, und eine spezifische Funktion wird ihnen meist abgesprochen. Mit dem Ziel, ein mögliches kortikales Repräsentationsareal der aurikulären Muskulatur aufzuspüren, wurde in der vorliegenden Untersuchung der primär motorische Kortex von 8 gesunden ProbandenInnen (6 männlich; durchschnittliches Alter: 26,5 Jahre) mittels transkranieller Magnetstimulation (TMS) untersucht (TMS-Mapping). Eine neuartige robotergestützte und neuronavigationsbasierte TMS-Technologie ermöglichte eine hochpräzise Spulenpositionierung (6 Freiheitsgrade) sowie eine randomisierte Stimulationsreihenfolge. Motorisch evozierte Potenziale (MEP) wurden mit Drahtelektroden am Musculus auricularis posterior (PAM) aufgezeichnet. Zum topographischen Vergleich wurde ein identischer Versuch für einen Handmuskel (FDI) durchgeführt.

Während der TMS-Untersuchungen des PAM und des FDI ließen sich in jeweils abgegrenzten Arealen über der Kopfoberfläche MEP ableiten. Hierzu war im PAM bei der Mehrzahl der VersuchsteilnehmerInnen eine Muskelvoraktivierung notwendig. Die aus den mittleren MEP-Amplituden der unterschiedlichen Stimulationspunkte berechneten Schwerpunkte (COGs) beider Muskeln befanden sich alle über dem Gyrus praecentralis. Die Entfernung zwischen den PAM und FDI COGs betrug im Mittel 26,3 mm, wobei die PAM COGs weiter lateral auf dem Gyrus praecentralis lokalisiert waren. 
In der vorliegenden Studie konnte erstmalig die kortikale Repräsentation der aurikulären Muskeln im Menschen identifiziert werden. Die Einbeziehung des primär motorischen Kortex in ihre Steuerung ist ein Indikator für eine erhaltene bzw. höhere Funktion dieser Muskeln im Menschen, welche noch weiter aufgedeckt werden muss. Darüber hinaus zeigte sich, dass alle Versuchsteilnehmerlnnen den PAM willkürlich, d. h. auf Aufforderung, aktivieren konnten. 


\section{Abkürzungen}

3D 3-dimensional

BOLD abhängig vom Blutsauerstoffgehalt (blood oxygen level dependent)

$\mathrm{cm}$ Zentimeter

COG Schwerpunkt (center of gravity)

EEG Elektroenzephalographie

EMG Elektromyographie

FDI Musculus interosseus dorsalis I

fMRT funktionelle Magnetresonanztomographie

FSL Oxford centre for functional MRI of the brain (FMRIB) software library

M. Musculus

MEP motorisch evozierte Potenziale

$\mathrm{mm}$ Millimeter

MRT Magnetresonanztomographie

ms Millisekunde

MSO maximale Stimulationsintensität (maximum stimulator output)

$\mathrm{mV}$ Millivolt

M1 primär motorischer Kortex

N. Nervus (Nerv)

PAM Musculus auricularis posterior (posterior auricular muscle)

PAMR Musculus-auricularis-posterior-Reflex (post-auricular muscle reflex)

s Sekunde

SMA supplementär motorischer Kortex

TMS transkranielle Magnetstimulation 


\section{EINLEITUNG}

\subsection{Die aurikuläre Muskulatur}

Bereits Charles Darwin mutmaßte seinerzeit über die Funktion und Evolution der aurikulären Muskeln. Er hatte festgestellt, dass wilde Tiere das Aufstellen der Ohren zur genauen Richtungsortung von Geräuschquellen nutzten, insbesondere bei drohenden Gefahren, während domestizierten Tieren diese Fähigkeit fehlte. Aufgrund seiner Beobachtungen spekulierte Darwin, dass domestizierte Tiere weniger Gefahren ausgesetzt gewesen seien, was zu einer Nichtnutzung der aurikulären Muskeln und infolgedessen zu ihrer Verkümmerung geführt habe (Darwin 1859).

Auch der Mensch verfügt über entsprechende aurikuläre Muskulatur, die sich in jedem Ohr aus acht verschiedenen Muskeln zusammensetzt. Sie sind unterteilt in intrinsische und extrinsische Bereiche. Während sowohl Ansatz als auch Ursprung der intrinsischen aurikulären Muskeln innerhalb der Ohrmuschel lokalisiert sind, befindet sich der Ursprung der extrinsischen aurikulären Muskeln außerhalb der Ohrmuschel am Schädelknochen (Henle 1858; Schwalbe 1897). Demzufolge ruft die Aktivierung der extrinsischen aurikulären Muskeln eine Bewegung der Ohrmuschel hervor.

Im Laufe des vergangenen Jahrhunderts konnten aufschlussreiche Entdeckungen an der humanen aurikulären Muskulatur verzeichnet werden. 1908 wurde erstmalig das okulo-aurikuläre Phänomen beschrieben, welches bei maximalem Blick zur Seite auftritt (Wilson 1908) und eine Aktivierung des intrinsischen Musculus auricularis transversus und damit ein Einwärtsrollen der Ohrmuschel be- 
wirkt (Heuser 1976; Schmidt und Thoden 1978; Urban et al. 1993). 1963 wurden schließlich Ausführungen über die Entdeckung des Musculus-auricularis-posterior-Reflex (PAMR) publiziert, der eine durch ein akustisches Signal ausgelöste (reflektorische) Aktivierung des hinteren Ohrmuskels beinhaltet und mit einer Latenz von ca. 10 ms nach dem akustischen Signal auftritt (Kiang et al. 1963; Cody und Bickford 1969; O’Beirne und Patuzzi 1999). Die verfügbaren Angaben bezüglich der Prävalenz des PAMR variieren zwischen $68-100 \%$ und sein Vorhandensein ist abhängig von der Art des akustischen Signals (Cody und Bickford 1969; O’Beirne und Patuzzi 1999; Purdy et al. 2005; Benning 2011).

Die Beteiligung der aurikulären Muskeln in okulo-aurikulärem Phänomen und PAMR deutet darauf hin, dass sie (insbesondere der Musculus auricularis transversus und der Musculus auricularis posterior), vergleichbar mit dem Musculus orbicularis oculi im Rahmen des Blinkreflexes, wenigstens teilweise durch Kerngebiete innerhalb des Hirnstamms kontrolliert werden (Hackley 1993; Kiziltan et al. 2010).

Beim Menschen wurde eine über die Ortung von Geräuschquellen hinausgehende Funktion dieser Muskeln bis heute nicht gefunden. Im Gegenteil, sie werden in der Regel als rudimentär beschrieben (Serra et al. 1986). In elektromyographischen (EMG) Studien konnte allerdings eine deutliche Aktivierung der extrinsischen aurikulären Muskeln, entweder als Co-Aktivierung im Rahmen unterschiedlicher Gesichtsausdrücke oder nach Aufforderung, die Ohrmuschel zu bewegen, nachgewiesen werden (Berzin und Fortinguerra 1993). Eine ausschließliche Aktivierung der aurikulären Muskulatur, d.h. ohne zeitgleiche Co-Aktivierung anderer mimischer Muskeln, zeigte sich jedoch bei keinem der Versuchsteilnehmerlnnen 
(Berzin und Fortinguerra 1993).

Ob die aurikulären Muskeln im Menschen auch auf kortikaler Ebene repräsentiert sind ist bisweilen nicht bekannt. Tract-Tracing-Studien nach zu urteilen, die an Rhesusaffen durchgeführt wurden, sind die aurikulären Muskeln auf kortikaler Ebene vor allem im supplementär motorischen (SMA) sowie im cingulären Kortex repräsentiert (Jenny und Saper 1987; Morecraft et al. 2001).

\subsection{Primär motorischer Kortex}

Der primär motorische Kortex (M1) befindet sich im Bereich der posterioren Wölbung des Gyrus praecentralis. Zusammen mit den supplementär motorischen, prämotorischen und posterioren parietealen Kortizes bildet er den sogenannten Motokortex, der zusammen mit subkortikalen Strukturen für die Steuerung, Planung und Ausführung von willkürlichen Bewegungen zuständig ist.

Über den Tractus corticonuclearis und den Tractus corticospinalis stehen einige Neurone des M1 (1. Motoneuron) direkt, d. h. monosynaptisch, mit (kontralateralen) motorischen Hirnnervenkernen und Vorderhornzellen des Rückenmarks (2. Motoneuron) in Verbindung. Dabei kreuzt der Großteil der kortikonukleären bzw. -spinalen Bahnen vor Verschaltung auf das 2. Motoneuron zur Gegenseite (Bartholow 1874).

Mitte des 20. Jahrhunderts wurden über elektrische Stimulation des M1 am wachen Patienten genauere Kenntnisse über die kortikale Repräsentation der einzelnen Körperregionen innerhalb des M1 erlangt (Penfield und Boldrey 1937). So 
zeigte sich eine somatotope Anordnung der kortikalen Repräsentationsareale im Sinne eines auf dem Kopf stehenden und größenverzerrten Homunkulus, sodass benachbarte Neuronenpopulationen jeweils in benachbarte Körperregionen projizieren (Penfield und Boldrey 1937). Die Größe der kortikalen Repräsentationsareale ist dabei nicht proportional zur Größe (Anzahl der Muskelfasern) der jeweiligen Muskeln. So sind z. B. die Repräsentationsareale Hand und Zunge im Vergleich zur Rumpfmuskulatur überproportional groß ausgedehnt.

\subsection{Transkranielle Magnetstimulation}

Die transkranielle Magnetstimulation (TMS) ist eine nicht-invasive Methode, um eine Stimulation von (kortikalen) Nervenzellen auszulösen (Barker et al. 1985). Seit ihrer Einführung hat sich die TMS im Rahmen neurologischer Diagnostik und neurowissenschaftlicher Fragestellungen etabliert.

Die Wirkweise der TMS basiert auf dem Prinzip der elektromagnetischen Induktion (Faraday 1832). Ein sich änderndes Magnetfeld erzeugt in einem elektrisch leitenden Medium eine elektrische Potenzialänderung. Der Stromfluss durch die Spule eines Magnetstimulators erzeugt dabei das Magnetfeld. Abhängig vom Magnetstimulator und der verwendeten Spule hat das erzeugte Magnetfeld typischerweise eine Dauer von 200-400 $\mu$ s und eine Flussdichte von ca. 1-3 Tesla (Weyh und Siebner 2007). Innerhalb eines bestimmten Areals wird durch das sich ändernde Magnetfeld ein elektrisches Feld erzeugt, welches bei Positionierung der TMS-Spule über kortikalen Nervenzellen und bei ausreichender Stimulationsintensität zu ihrer Depolarisation führen kann. An welchem genauen Ort innerhalb einer Nervenzelle der Erregungsursprung stattfindet, ist nicht genau bekannt. Die 
niedrigste Erregungsschwelle wurde für Neurone, die erst parallel zum elektrischen Feld verlaufen und sich später biegen gezeigt (Amassian et al. 1992; Maccabee et al. 1993; Kobayashi und Pascual-Leone 2003). Auf Grund der physikalischen Eigenschaften des durch TMS induzierten elektrischen Feldes, dessen Stärke mit zunehmender Distanz zur Spule abnimmt, wurde lange Zeit vermutet, dass die neuronale Aktivierung an der Konvexität der Gyri, an Enden und Biegungen von kortikalen (und subkortikalen) Axonen stattfinde (Day et al. 1989). Neuere Forschungsergebnisse weisen jedoch darauf hin, dass die neuronale Aktivierung eher tiefer im Sulcus erfolgt, da hier auf Grund des Faserverlaufs der Neurone die effektive Stromstärke trotz der tiefer liegenden Lokalisation am größten ist (Fox et al. 2004; Laakso et al. 2014). Insgesamt werden durch TMS alle im Bereich des elektrischen Feldes erregbaren Zellen gleichermaßen stimuliert. Durch die Anwendung von unterschiedlichen Stimulationsintensitäten und -Protokollen kann jedoch, abgängig von Lokalisation und Beschaffenheit einzelner Nervenzellpopulationen, eine relative Selektivität in Bezug auf ihre Stimulation hergestellt werden (Kobayashi und Pascual-Leone 2003; Ridding und Rothwell 2007). So wurde bereits gezeigt, dass niedrige Stimulationsintensitäten eher zu einer Aktivierung von Interneuronen und höhere Stimulationsintensitäten zu einer direkten Aktivierung des 1. Motorneurons führen (Di Lazzaro et al. 1998; Rusu et al. 2014).

Alternativ zur TMS können Nervenzellen auch direkt elektrisch, d. h. ohne den Umweg über die Induktion eines Magnetfeldes, transkraniell stimuliert werden (Merton und Morton 1980). Im Vergleich zur direkten elektrischen Stimulation hat jedoch die Nutzung eines Magnetfeldes zahlreiche Vorteile. Im Gegensatz zu elektrischem Strom breitet sich ein Magnetfeld problemlos durch biologisches Gewebe aus (Weyh und Siebner 2007). Dies bedeutet, dass bei der TMS aufgrund 
des geringeren Widerstandes relativ geringere Stimulationsintensitäten für eine Stimulation von Nervenzellen notwendig sind. Darüber hinaus ist ein direkter Kontakt zwischen Kopfoberfläche und TMS-Spule, z. B. über auf der Haut platzierte Elektroden, für die TMS nicht notwendig. Weiterhin führt die TMS nur zu einer sehr schwachen Reizung von Schmerzrezeptoren innerhalb der Haut sowie Muskelfasern, sodass ihre Durchführung eher schmerzarm ist und auf Grund dessen in der Regel gut toleriert wird (Bischoff et al. 1993; Ridding und Rothwell 2007).

Wegen der guten Verträglichkeit bei einem insgesamt positiven Nebenwirkungsprofil wurde die TMS seit ihrer Einführung in vielfältiger Hinsicht weiterentwickelt und ergänzt. So kommen je nach Fragestellung unterschiedliche Spulentypen (z.B. Rundspulen, Schmetterlingsspulen oder achtförmige Spulen) und TMS-Protokolle zum Einsatz (z. B. Einzelpuls-, repetitive und paired-pulse TMS).

Heute sind die Anwendungsgebiete der TMS breit gefächert. Neben einer vielfältigen Anwendung im Forschungsbereich findet die TMS einen klinischen Nutzen im Feld der neurophysiologischen Diagnostik. Ein therapeutischer Einsatz im Rahmen der Therapie neurologischer und psychiatrischer Erkrankungen wurde bereits in verschiedenen Studien geprüft. Hier scheint sich unter anderem ein positiver Effekt von repetitiver TMS auf depressive Störungen sowie auf die Rehabilitation nach ischämischem Schlaganfall abzuzeichnen (McNamara et al. 2001; Kobayashi und Pascual Leone 2003; Mansur et al. 2005; George et al. 2010).

\subsection{Motorisch evozierte Potenziale und Elektromyographie}

Die häufigste Anwendung der TMS beinhaltet die Ableitung motorisch evozierter 
Potenziale (MEP), d. h. Summenaktionspotenzialen in einem bestimmten Muskel nach Stimulation des primär motorischen Kortex (Rossini et al. 1994; Rothwell et al. 1999). Zur Ableitung von MEP wird die TMS-Spule über dem jeweiligen Repräsentationsareal des untersuchten Muskels positioniert und eine überschwellige (siehe unten) Stimulation durchgeführt, die eine Erregung von kortikalen Neuronen (1. Motoneuron oder Interneurone) zur Folge hat. Nach Verschaltung auf das 2. Motoneuron kommt es zu einer Aktivierung der entsprechenden (durch das 2. Motoneuron innervierten) Muskelfasern (Amassian et al. 1987; Ridding und Rothwell 2007), die als kurzzeitige Potenzialänderung mittels EMG aufgezeichnet werden kann. Zur Ableitung von Muskelpotenzialen können sowohl auf der Haut platzierte Oberflächenelektroden als auch im Muskel platzierte Nadel- oder Drahtelektroden verwendet werden.

Bei der Analyse der ausgelösten MEP wird ein Augenmerk auf die Latenz, peakto-peak-Amplitude, Fläche, Morphologie sowie, bei Vorspannung des jeweiligen Muskels, die Dauer der kortikalen Innervationsstille (cortical silent period) gelegt. Im Rahmen der diagnostischen Anwendung der TMS können Abweichungen innerhalb der genannten Parameter einen Hinweis auf unterschiedliche zentrale und periphere Leitungsstörungen liefern (Kobayashi und Pascual-Leone 2003). Der Einfluss des Untersuchers auf die TMS-Ergebnisse sollte möglichst minimiert werden. Dabei birgt die Spulenpositionierung großes Fehlerpotenzial . Da bereits kleine Unterschiede in Bezug auf die Ausrichtung der TMS-Spule einen deutlich messbaren Effekt auf MEP-Amplituden haben (Mills et al. 1992; Richter et al. 2013a), muss die Spule bei jeder Messung identisch positioniert werden. Sie wird tangential zur Kopfoberfläche gehalten und in einem bestimmten Rotationswinkel ausgerichtet. Zur Stimulation des Handareals hat sich eine postero-laterale Spu- 
lenorientierung mit einer Rotation in der Saggitalebene um ca. $45^{\circ}$ als am besten geeignet (niedrigste Stimulationsschwelle) herausgestellt (Mills et al. 1992). Bezüglich der optimalen Spulenposition zur Stimulation der unterschiedlichen Gesichtsmuskel-Repräsentationsareale finden sich in der Literatur widersprüchliche Angaben (Guggisberg et al. 2001; Dubach et al. 2004). Auch der Druck, der durch die Spule auf die Kopfoberfläche ausgeübt wird, muss konstant gehalten werden. Diesbezüglich wurde gezeigt, dass Druckunterschiede zu erheblichen Potenzialänderungen des induzierten elektrische Feldes führen (Richter et al. 2013b).

Die MEP-Amplituden, welche die Amplitude zwischen der positiven und negativen Potenzialänderung darstellen (peak-to-peak), sind innerhalb eines Individuums auch bei identischen Stimulationsbedingungen und innerhalb einer Sitzung in hohem Maße variabel (Hess und Ludin 1988; Brasil-Neto et al. 1992; Wassermann et al. 2002). Zur Reduzierung der Varianz sollten dementsprechend mehrere Einzelmessungen durchgeführt werden (Hess und Ludin 1988; Ellaway et al. 1998). Die Ursachen für das hohe Maß an Variabilität sind nicht genau bekannt, am ehesten wird sie durch Unterschiede innerhalb der kortikalen Erregbarkeit hervorgerufen (Kiers et al. 1993). Dafür spricht, dass die Amplitudenvariabilität bei steigender Stimulationsintensität und Muskelvoraktivierung ab- (Kiers et al. 1993; Brasil-Neto et al. 1992) und bei steigender Entfernung vom optimalen Stimulationsort (sog. hotspot; s. u.) zunimmt (Brasil-Neto et al. 1992). Auch die im Laufe einer TMSSitzung auftretenen Veränderungen des Wachheitszustandes des Probanden beeinflussen die MEP-Amplituden (Ziemann et al. 1999; Lang et al. 2011). Neben MEP-Amplituden sind auch ihre Latenzen, d. h. die Dauer zwischen Stimulus und Beginn des MEP, von den Stimulationsbedingungen abhängig. So sind bei steigender Stimulationsintensität und Muskelvoraktivierung die Latenzen verkürzt und 
ihre Standardabweichungen geringer (Brasil-Neto et al. 1992; Rossini et al. 1987). Am optimalen Stimulationsort (sog. hotspot) sind die Latenzen jeweils am kürzesten (Rossini et al. 1994).

Die silent period beschreibt ein durch TMS hervorgerufenes vorübergehendes Verschwinden oder eine deutliche Minderung der EMG-Aktivität bei tonischer Muskelaktivierung. Sie wird vorrangig durch kortikale Inhibition der 1. Motorneurone durch Interneurone, im Anschluss an deren Aktivierung durch TMS, hervorgerufen (Roick et al. 1993; Wilson et al. 1993; Rossini et al. 1994). Die Dauer der silent period variiert zwischen unterschiedlichen Muskeln (Roick et al. 1993), ist abhängig von der Konfiguration des TMS-Pulses und korreliert positiv mit MEP-Amplitude und Stimulationsintensität (Orth und Rothwell 2004).

\subsection{Hotspot und Stimulationsschwelle (threshold)}

Für jeden Muskel gibt es einen bestimmten Ort über der Kopfoberfläche, der die optimale Stimulationsposition darstellt, den sogenannten hotspot. Er ist definiert als jener Punkt, an welchem die Stimulationsschwelle am niedrigsten und die MEP-Latenzen am kürzesten sind (Rossini et al. 1994). Die Stimulationsschwelle ist definiert als die Intensität, bei der durch Stimulation am hotspot in $50 \%$ der Messungen (10-20 Wiederholungen) MEP-Amplituden von $\geq 50 \mu \mathrm{V}$ abgeleitet werden (Rossini et al. 1994; Rothwell et al. 1999). Zur Erhebung der Stimulationsschwelle wurden unterschiedliche Verfahren publiziert (Rothwell et al. 1999; Awiszus 2003). Die Stimulationsschwellen variieren zwischen Individuen und untersuchten Muskeln, sind jedoch, im Gegensatz zu den stark variablen MEP-Amplituden, im einzelnen Probanden relativ konstant (Malcolm et al. 2006; Lang et al. 2011). Die längsten 
silent periods wurden nicht ausschließlich am hotspot beobachtet (Wassermann et al. 1993).

\subsection{Mapping von kortikalen motorischen Repräsentationsarealen mittels transkranieller Magnetstimulation}

Im Rahmen der Erforschung und Lokalisation von kortikalen Repräsentationsarealen unterschiedlicher Muskeln hat sich die TMS seit ihrer Einführung etabliert (Wilson et al. 1993). Hier bietet das TMS-Mapping die Möglichkeit, eine Kartierung der motorischen Repräsentationsareale durchzuführen. Dabei werden aufgrund der höheren Fokalität meist achtförmige Spulen angewendet (Lotze et al. 2003; Malcolm et al. 2006; Weyh und Siebner 2007). Ziel einer Mapping-Untersuchung ist, den Kortex nach dem hotspot des jeweiligen untersuchten Muskels abzusuchen sowie die Ausdehnung eines Repräsentationsareals zu erforschen. Hierzu wird die Spule an unterschiedlichen Punkten auf der Kopfoberfläche positioniert und eine überschwellige Stimulation durchgeführt. Im Allgemeinen werden dazu Stimulationsintensitäten von 110 - 120\% oberhalb der Stimulationsschwelle verwendet (Lotze et al. 2003; Malcolm et al. 2006). Zeitgleich erfolgt die Ableitung von MEP im untersuchten Muskel. Die Stimulationspunkte sind meist als Raster oder kreuzförmig angeordnet (Wassermann et al. 1992; Rödel et al. 2000). Bezüglich des genauen Ablaufs einer solchen TMS-Mapping-Untersuchung finden sich in der Literatur divergierende Angaben mit vielen unterschiedlichen Protokollen und Prozeduren. Der Abstand der Rasterpunkte ist in der Regel genau festgelegt und variiert bei den betrachteten Studien in der Regel zwischen einem und 2 Zentimetern (Wassermann et al. 1994; Malcolm et al. 2006; Littmann et al. 2013). Auch die Anzahl der TMS-Pulse pro Rasterpunkt ist heterogen, in der 
Regel werden zwischen 3 und 6 Stimuli pro Punkt verwendet (Lotze et al. 2003; Sparing et al. 2008). Die Abfolge der Stimuli erfolgt aus praktischen Gründen in einer festgelegten Reihenfolge, wobei die einzelnen Rasterpunkte entweder abwechselnd (Lotze et al. 2003) oder mehrfach hintereinander (Malcolm et al. 2006; Littmann et al. 2013) stimuliert werden. Zwischen den einzelnen TMS-Pulsen sollte ein Abstand von wenigstens 5 Sekunden liegen um Effekte auf das folgende MEP zu vermeiden (Kiers et al. 1993). MEP können unter Ruhebedingungen oder Vorspannung abgeleitet werden (Wassermann et al. 1994). Zur Darstellung der Mapping-Ergebnisse werden Mittelwert oder Median der MEP-Amplituden, die maximale Amplitude oder die MEP-Fläche ( $\mathrm{mV}$ x ms) in ein Koordinatensystem eingetragen. Teilweise wird daraus eine Karte (topographic map) erstellt, indem die betrachteten Parameter grafisch dargestellt werden. Darüber hinaus kann aus den Daten ein Schwerpunkt (center of gravity: COG) errechnet werden, welcher den optimalen Stimulationsort darstellt (Wassermann et al. 1992). Die Lokalisation der COGs ist unabhängig von Schwankungen der kortikospinalen Erregbarkeit, d. h. unabhängig von der Variabilität der MEP-Amplituden, und zeigt eine hohe Konsistenz über verschiedene TMS-Sitzungen (Weyh und Siebner 2007). Im Vergleich mit funktioneller Bildgebung mittels fMRT zeigt sich eine hohe Korrelation der COGs beider Methoden, wenngleich die TMS-COGs einige Millimeter bis wenige Zentimeter weiter anterior lokalisiert sind als jene, die durch fMRT ermittelt wurden (Herwig et al. 2002; Lotze et al. 2003).

\subsection{Spulenpositionierung und Neuronavigation}

Die Durchführung von TMS-Mapping-Untersuchungen wurde in den vergangenen Jahren in vielfältiger Weise weiterentwickelt. Hierbei wurde insbesondere die Aus- 
fürung der Spulenpositionierung mit dem Ziel des Erreichens einer höheren Genauigkeit und Reliabilität auf unterschiedliche Art und Weise überarbeitet.

Das konventionelle TMS-Mapping beinhaltet eine Markierung der Stimulationspunkte (z. B. in Form eines Rasters) auf der Kopfoberfläche oder einer Kappe, die auf dem ProbandInnen-Kopf befestigt ist (Wassermann et al. 1992). Über den einzelnen Zielpunkten erfolgt die Platzierung der Spule manuell durch den Untersucher tangential, mit konstantem Druck und in einem festgelegten Rotationswinkel zur Kopfoberfläche. Die Rastergröße variiert im Allgemeinen zwischen 5x5 und 7x7 cm (Malcolm et al. 2006; Guerra et al 2014).Bei ausreichender Größe des Rasters hat diese Methode den Vorteil, dass sie eine unvoreingenommene und ergebnisoffene Suche nach einem möglichen kortikalen Repräsentationsareal ermöglicht. Dies ist insbesondere notwendig, wenn noch keine Kenntnisse über das untersuchte Repräsentationsareal, z.B. durch andere bildgebende Verfahren, vorhanden sind. Nachteile dieser Methode sind, dass das Repräsentationsareal komplett durch das Raster abgedeckt sein sollte, das in Folge dessen eine ausreichende Größe aufweisen sowie präzise positioniert werden muss. Der Untersucher muss dementsprechend bereits über Kenntnisse über den ungefähren Ort des Repräsentationsareals verfügen, z. B. durch eine vorherige manuelle Suche (Lotze et al. 2003; Sparing et al. 2008). Weiterhin muss zur Festlegung der Stimulationsintensität die Stimulationsschwelle bereits im Vorfeld ermittelt werden (Malcolm et al. 2006).

Deskriptiv werden die Ergebnisse des TMS-Mappings üblicherweise in das 10-20-System (Jasper 1958) eingeordnet, welches auch bei der Positionierung von EEG-Elektroden Anwendung findet. Die individuell variable Kortexanatomie wird bei dieser Methode nicht berücksichtigt. Daraus folgt, dass sich im Vergleich 
zwischen einem definierten Punkt auf der Kortexoberfläche und seinem Äquivalent innerhalb des 10-20-Systems zwischen den einzelnen Individuen Streuungen von ca. 20 mm ergeben (Herwig et al. 2003). Alternativ können die TMS-Mapping-Ergebnisse bei bekannter Lokalisation der Rasterpunkte (z.B. innerhalb eines Koordinatensystems) auf die Kortexoberfläche (MRT-Daten) projiziert werden (Kantelhardt et al. 2010).

Bei bekannter Lokalisation des kortikalen Zielareals kann die Spulenposition, als Alternative zu einem auf der Kopfoberfläche oder Kappe markierten Raster oder der Verwendung von anatomischen Landmarken innerhalb des 10-20-Systems, auch an der individuellen Kortexanatomie ausgerichtet werden. Hierzu wurden in den vergangenen Jahren unterschiedliche, vor allem neuronavigationsbasierte Systeme entwickelt und präsentiert. Dazu werden TMS-Spule und individuelle Kortexanatomie (MRT-Daten) innerhalb eines Koordinatensystems registriert, visualisiert und die Stimulationspunkte anhand der individuellen Kortexanatomie festgelegt (Krings et al. 1997; Ettinger et al. 1998). Dieses Vorgehen hat einen höheren Grad an Reliabilität und Präzision in Bezug auf die Position der TMS-Spule (Gugino et al. 2001; Sparing et al. 2008) und der Mapping Ergebnisse (Julkunen et al. 2008; Weiss et al. 2012).

Um den verbleibenden Einfluss des Untersuchers durch manuelle Spulenpositionierung auf die TMS-Mapping-Ergebnisse zu minimieren wurden kürzlich Robotersysteme eingeführt, die eine exakte, teilweise automatisierte und untersucherunabhängige Ansteuerung der Zielpunkte ermöglichen (Kantelhardt et al. 2010; Richter et al. 2013a/b). 


\section{$1.8 \quad$ Ziele der Untersuchung}

Da die Neuroanatomie der humanen aurikulären Muskeln bis heute unbekannt ist, zielt die vorliegende Studie auf die Suche, Identifizierung und Lokalisierung eines möglichen kortikalen Repräsentationsareals. Hierzu wurde mittels TMS ein Mapping des primär motorischen Kortex durchgeführt.

Eine Repräsentation innerhalb kortikaler Bereiche, die an der Ausführung und Planung von Willkürbewegungen beteiligt sind, wie z.B. der primär-, oder sekundär motorische Kortex sowie derSMA (Rao etal. 1993), würde einen Unterschied bezüglich der Neuroanatomie der aurikulären Muskulatur zwischen Menschen und dem Rhesusaffen aufzeigen und Grund zu derAnnahme geben, dass die Funktion der aurikulären Muskeln im Menschen über die Lokalisation von Geräuschquellen hinaus ginge.

Ein weiteres Ziel der vorliegenden Untersuchung ist den Einfluss des Untersuchers und die Variabilität von MEP auf die Mapping-Ergebnisse zu minimieren. Dazu wurde eine neuartige hochpräzise robotergestützte Technologie zur Spulenpositionierung implementiert und die Stimulationsreihenfolge erstmalig komplett randomisiert.

Da TMS-Mapping innerhalb der Forschung eine breite Anwendung findet, könnte eine Weiterentwicklung des Verfahrens, insbesondere eine Minimierung des Einflusses des Untersuchers und der MEP-Variabilität auf die Mapping-Ergebnisse, die Qualität unterschiedlichster TMS-Studien positiv beeinflussen. 


\section{MATERIAL UND METHODEN}

Mittels eines roboter- und bildgestützten, neuronavigierten TMS-Mapping-Verfahrens wurde in der vorliegenden Studie an 8 gesunden ProbandInnen der M1 hinsichtlich eines möglichen kortikalen Repräsentationsareals des Musculus auricularis posterior (PAM) und zum topographischen Vergleich des Musculus interosseus dorsalis I (FDI) untersucht.

\subsection{Versuchsgruppe}

Die Gruppe der insgesamt 8 Versuchsteilnehmerlnnen setzte sich aus 6 Männern und 2 Frauen zusammen. Alle ProbandInnen waren gesund und im Alter zwischen 21 und 38 Jahren (Mittelwert: 26,5). Dem Edinburgh-Händigkeitstest (Oldfield 1971 ) entsprechend waren alle ProbandInnen Rechtshänder. Nach ausführlicher Aufklärung über den Versuchsablauf und dessen Risiken wurde von jeder/jedem Versuchsteilnehmerln eine schriftliche Einverständniserklärung eingeholt. Die Studie wurde durch die Ethikkommission der Georg-August-Universität Göttingen genehmigt und steht im Einklang mit der Deklaration von Helsinki (www.wma.net).

\subsection{Versuchsdurchführung}

Die TMS-Mapping-Experimente bestanden aus jeweils 4 Einzeluntersuchungen. Die ersten zwei Untersuchungen betrafen den FDI, die beiden folgenden Sitzungen den PAM. Während der Experimente saßen die Versuchsteilnehmerlnnen in einer bequemen Position auf einem Zahnarztstuhl. Der Kopf war angelehnt und nicht fixiert. Als Hörschutz wurden Ohrenstöpsel verwendet. Die TMS wurde mit 
einem Magstim 200 Magnetstimulator mit einem maximalen magnetischen Feld von 2,2 Tesla und einer 8-förmigen Magnetspule mit einem Windungsdurchmesser von 70 mm durchgeführt (Magstim Company, Whitland, UK). Für jeden TMS-Stimulus wurde die Spule tangential über der Kopfoberfläche positioniert. Die Spule war in postero-lateraler Richtung mit einem Rotationswinkel von $45^{\circ}$ ausgerichtet. Zur exakten Spulenpositionierung wurde ein vollautomatisierter Roboterarm verwendet (siehe unten). Das minimale Interstimulusintervall betrug 5 Sekunden. Abhängig von der Dauer, die der Roboterarm für die Bewegung zwischen den einzelnen Rasterpunkten beanspruchte, zählte das tatsächliche Interstimulusintervall zwischen 5 und 8 Sekunden. Aus Sicherheitsgründen führte der Roboterarm Bewegungen in einer relativ niedrigen Geschwindigkeit durch.

Die TMS-Untersuchung des FDI wurden während vollkommener Muskelentspannung durchgeführt. Die Aufzeichnungen der MEP erfolgte mittels Oberflächenelektroden $(\mathrm{Ag}-\mathrm{AgCl})$ mit der aktiven Elektrode über dem Muskelbauch und der Referenzelektrode über der Medialseite des proximalen Interphalangealgelenks des Indexfingers. Das Signal wurde verstärkt, zwischen 2 und $2000 \mathrm{~Hz}$ Breitband gefiltert (Digitimer D360, Digitimer Ltd.), mit 5000 Hz von einem A/D-Wandler digitalisiert (CED micro1401 mkII, Cambridge Electronic Design (CED) Ltd, Cambridge, UK) und auf einem Standard-PC aufgezeichnet. Die Analyse wurde in Echtzeit durch ein eigenes Signal-Softwareskript (CED Signal v4.08) durchgeführt (Hewitt 2011). Bei fehlender Muskelentspannung wurden die einzelnen Messungen automatisch wiederholt.

Vor den TMS-Untersuchungen des PAM wurde jede/ jeder ProbandIn auf die Fähigkeit, den PAM nach Aufforderung zu aktivieren, getestet. Hierzu wurden die 
Versuchsteilnehmerlnnen gebeten, die Ohrmuschel so stark wie möglich nach hinten zu bewegen. Zur Aktivierungskontrolle wurde innen zeitgleich das EMGRohsignal auf einem Monitor sowie ein akustisches Feedback präsentiert. Die Übung verfolgte das Ziel, eine EMG-Aktivierung des PAM nach Aufforderung wiederholbar zu machen. Jene Versuchsteilnehmerlnnen, die auf Aufforderung keine klar abgrenzbaren EMG-Signale produzieren konnten, wurden in einer weiteren EMG-Sitzung dazu angeleitet (3 ProbandInnen). Sie wurden aufgefordert, verschiedene Bewegungen der mimischen Muskulatur auszuführen, wie z.B. lachen oder die Stirn runzeln. Zeitgleich wurde das PAM-EMG-Signal auf einem Monitor präsentiert. Ziel der Sitzung war es, dass die VersuchsteilnehmerInnen lernten, durch welche mimische Bewegung sie reproduzierbare EMG-Signale im PAM erzeugten.

Um eine Beeinflussung und ggf. Verfälschung der MEP durch den PAMR auszuschließen, wurden alle Probandlnnen auf das Vorhandensein des PAMR getestet. Dazu wurde vor der ersten TMS-Sitzung ein Kontrollexperiment durchgeführt, in welchem das Geräusch des TMS-Pulses präsentiert wurde, ohne dass eine Stimulation des Kortex erfolgte (Positionierung der Spule vertikal zur Kopfoberfläche).

Für die EMG-Aufzeichnungen im PAM wurden Drahtelektroden mit einem Durchmesser von $50 \mu \mathrm{m}$ verwendet. Die Elektroden sollten die EMG-Signalqualität erhöhen und eine Beeinflussung der EMG-Signale durch Aktivierung entfernter fazialer Muskulatur vermeiden (Mangun et al. 1986). Die aktive Elektrode wurde direkt innerhalb des PAM und die Referenzelektrode subkutan in der Mitte der Hinterseite der Ohrmuschel platziert (Abbildung 1). Unter dieser Elektrodenanordnung konnten in vorangegangenen Experimenten die höchsten und reproduzier- 
barsten EMG-Signale im PAM aufgezeichnet werden (O’Beirne und Patuzzi 1999). Das TMS-Mapping des PAM wurde während Muskelvorspannung durchgeführt. Um die ProbandInnen zu erinnern, die Ohrmuschel nach hinten zu ziehen, wurde $1 \mathrm{~s}$ vor dem TMS-Puls ein akustisches Signal präsentiert. Zur Kontrolle der Voraktivierung wurde die EMG-Amplitude innerhalb der 200 ms vor dem TMS-Stimulus während des Experiments kontrolliert. Im Falle einer nicht durchgeführten Voraktivierung wurde die Messung automatisch ausgemustert und im Laufe des Experiments wiederholt.

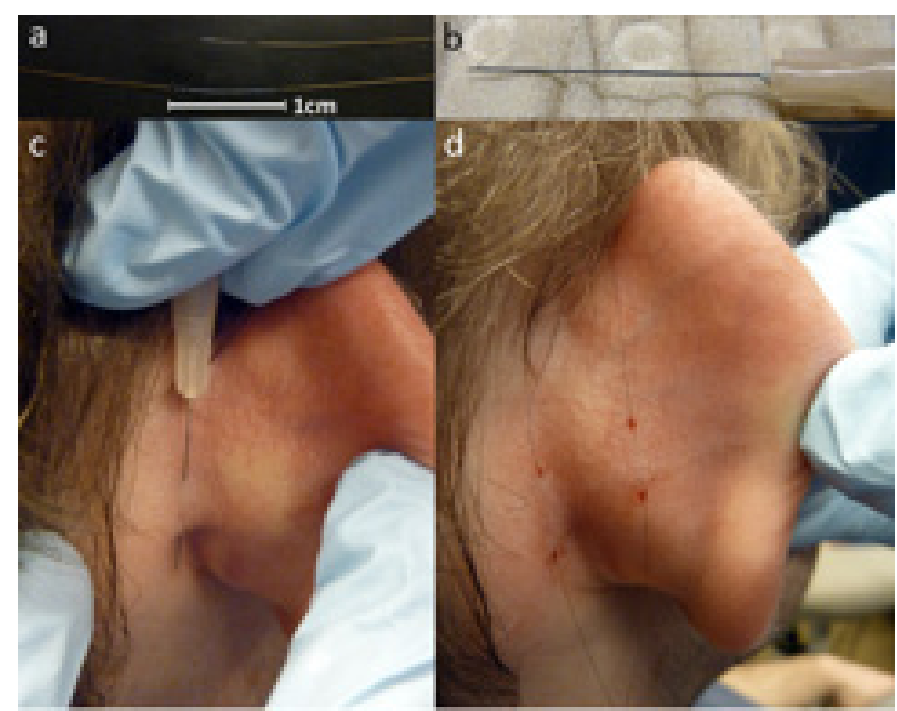

Abbildung 1: Elektromyographische Ableitung von Muskelpotentialen des Musculus auricularis posterior über Drahtelektroden

10-cm-messende Drahtelektrode mit einem Durchmesser von $50 \mu \mathrm{m}$. Die Isolierung wurde an beiden Enden sowie in der Mitte der Elektrode über jeweils $1 \mathrm{~cm}$ entfernt (a). Drahtelektrode innerhalb einer Hohlnadel (b). Nach zweimaligem Durchstechen der Haut verbleibt die Elektrode nach Entfernung der Hohlnadel in situ. Der mittlere, unisolierte Teil der Elektrode befindet sich unterhalb der Hautoberfläche (c). Beide Elektroden in situ mit der aktiven Elektrode innerhalb des PAM (links) und der Referenzelektrode im Zentrum der hinteren Ohrmuschel (rechts) (d).

\subsection{Roboter- und bildgestützte transkranielle Magnetstimulation}

Um die Genauigkeit und Reliabilität der Spulenposition zu erhöhen, wurde in der vorliegenden Studie eine Roboter- und bildgestützte TMS-Mapping-Technologie verwendet, welche aus einem Adept Viper s850 Roboterarm (Adept Technology, Inc. Livermore, CA, USA) in Kombination mit einem NDI Polaris Vicra Infrarot-Kamerasystem zur Positionsbestimmung (Positionsfehler: $\pm 0.5 \mathrm{~mm}$ ) bestand (Kantelhardt et al. 2010). Eine spezielle Roboternavigationssoftware (ANT, Enschede, 
Netherlands) steuerte den Roboterarm, welcher die Positionierung der TMS-Spule durchführte. Für jede/n Versuchsteilnehmerln erzeugte die Software aus individuellen MRT-Daten des Schädels, die im Vorfeld an die Experimente in einem 3-Tesla-MRT-Scanner (Magnetom Trio, Siemens, Erlangen, Germany) erzeugt wurden, ein 3D-Modell der Kopfoberfläche. Die Software war darüber hinaus für die Erstellung der Stimulationspunkte und die Echtzeitanalyse der Kopfposition im Raum zuständig, sodass Kopfbewegungen durch kompensatorische Bewegungen des Roboterarms ausgeglichen wurden. Auf die Kopfoberfläche bezogen, wurde die Spule jeweils tangential in $45^{\circ}$ postero-lateraler Ausrichtung über jedem Zielpunkt (Positionsfehler: $\pm 0.02 \mathrm{~mm}$ ) positioniert. Die Stimulationsreihenfolge war über alle Punkte und für jeden einzelnen TMS-Puls randomisiert und wurde durch das Signal-Softwareskript festgelegt (Hewitt 2011). Selbiges Softwareskript führte auch eine Echtzeitanalyse in Bezug auf das Vorhandensein von Muskelvorspannung durch.

Für jeden Muskel wurde, beginnend mit dem FDI, ein zweistufiges TMS-MappingProtokoll, welches jeweils zwei Sitzungen umfasste, durchgeführt. Die Stimulationspunkte waren rasterförmig auf der Kopfoberfläche angeordnet. Ein Raster setzte sich aus 49 Zielpunkten zusammen ( $7 \times 7$ ). Der Abstand zwischen den Stimulationspunkten betrug jeweils $10 \mathrm{~mm}$. Um das Raster optimal auszurichten und die Stimulationsintensität für das erste Mapping-Experiment festzulegen, wurde im Vorfeld ein vorläufiger FDI-hotspot bestimmt. Die vorläufige hotspot Suche bestand in einer manuellen Suche nach dem Punkt auf der Kopfoberfläche, durch dessen Stimulation die höchsten MEP-Amplituden bei niedrigster Stimulationsintensität ausgelöst wurden. An diesem Punkt wurde mit Hilfe eines wahrscheinlichkeitsbasierten Algorithmus dreimalig eine vorläufige Ruhemotorschwelle bestimmt 
(Awiszus 2003). Für das erste Mapping-Experiment wurde das Rasterzentrum auf den vorläufigen hotspot ausgerichtet, die Stimulationsintensität auf $120 \%$ der mittleren vorläufigen Ruhemotorschwelle festgelegt und jeder Rasterpunkt 6-mal stimuliert. Nach dem ersten Mapping-Experiment wurde aus den MEP-Amplituden das COG kalkuliert (siehe unten) und an diesem Punkt erneut dreimalig die Ruhemotorschwelle bestimmt. Das COG der ersten Sitzung diente schließlich als Mittelpunkt des Rasters für die zweite Sitzung. Dieses Protokoll stellte sicher, dass das kortikale Repräsentationsareal in der Mitte des Rasters lokalisiert war und komplett vom Raster umschlossen wurde. Für das finale Mapping wurde die Stimulationsintensität auf $120 \%$ der (neuen) mittleren Ruhemotorschwelle festgelegt und die Anzahl der Stimuli pro Punkt auf 12 erhöht. Nach der zweiten Sitzung wurde erneut ein COG berechnet und eine finale Ruhemotorschwelle ermittelt.

Um die kortikale PAM-Repräsentation mittels TMS zu lokalisieren, wurde ein ähnliches zweistufiges Protokoll durchgeführt. Für die erste Sitzung wurde das Raster aus der zweiten FDI-Sitzung um jeweils $2 \mathrm{~cm}$ anterior und lateral verschoben und die Stimulationsintensität auf $140 \%$ der FDI-Ruhemotorschwelle festgelegt. Die erste PAM-Mapping-Sitzung beinhaltete 6 Stimuli pro Punkt. Aus den Daten wurde ein vorläufiges COG berechnet. Wegen der Muskelvoraktivierung während der TMS-Untersuchung des PAM konnte für diesen Muskel keine Ruhemotorschwelle bestimmt werden. Aus diesem Grund wurde ein anderes Protokoll angewandt, um die Stimulationsintensität für das finale Mapping-Experiment festzulegen. Dieses Protokoll bestand in der Durchführung einer intensitätsabhängigen (zwischen 10 und $100 \%$, in 10er Schritten, 12 Pulse pro Intensität) MEP-Rekrutierung über dem COG der ersten Mapping-Sitzung des PAM. Für die zweite und finale PAM-Sitzung wurde das Raster über dem COG der ersten Sitzung zentriert und die Stimulati- 
onsintensität oberhalb des Wendepunkts der Rekrutierungskurve verwendet. Am aus den Daten der zweiten Sitzung berechneten COG wurden schließlich zwei weitere Rekrutierungskurven aufgezeichnet, erstere während und eine weitere ohne Muskelvoraktivierung.

\subsection{Datenanalyse}

Die Datenanalyse wurde mit Matlab (MathWorks, Natick, MA) durchgeführt. Die MEP-Amplitude an jedem Stimulationspunkt (Abbildungen 2 und 3) wurden zur Berechnung der COGs und der topographischen Karten herangezogen (Wassermann et al. 1992). Die topographic maps wurden berechnet, indem jede Amplitude relativen Werten zugeordnet wurde, wobei die Skalierung an Hand der minimalen und maximalen Amplituden ausgerichtet war. Bei fehlender Voraktivierung betrug die minimale Amplitude während der FDI-Experimente nahezu $0 \mu \mathrm{V}$. Im Gegensatz dazu führte die Muskelvorspannung im PAM zu einer starken Variabilität der EMG-Grundaktivität innerhalb des Zeitintervalls, in dem sich die MEP befanden und das zur Berechnung der Karten herangezogen wurde. Um den Einfluss der Muskelvorspannung auf die Amplituden zu minimieren, d. h. zur Erhöhung des Signal/Rausch-Verhältnisses wurde ein Cut-Off eingeführt:

$$
v_{i^{*}}=v_{i}-v_{\min }-\left(v_{\max }-v_{\min }\right) / 2
$$

Ausgehend von diesen Daten wurden die COGs mit folgender Formel berechnet:

$$
\text { COG }=\Sigma v_{i^{*}} x / \Sigma v_{i^{*}}, \Sigma v_{i^{*}} y / \Sigma v_{i^{*}} ; \text { für alle } v_{i^{*}}>0
$$



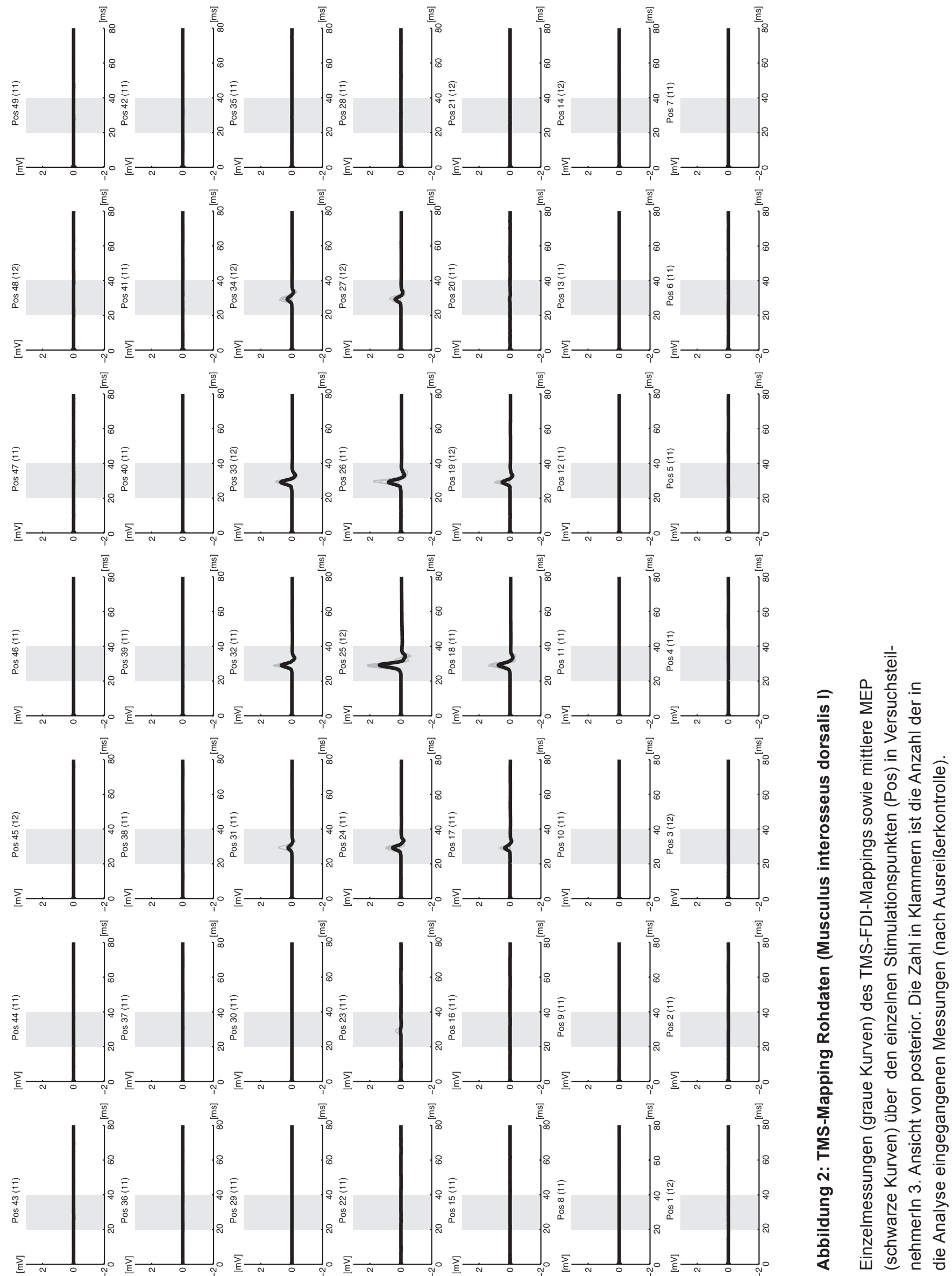

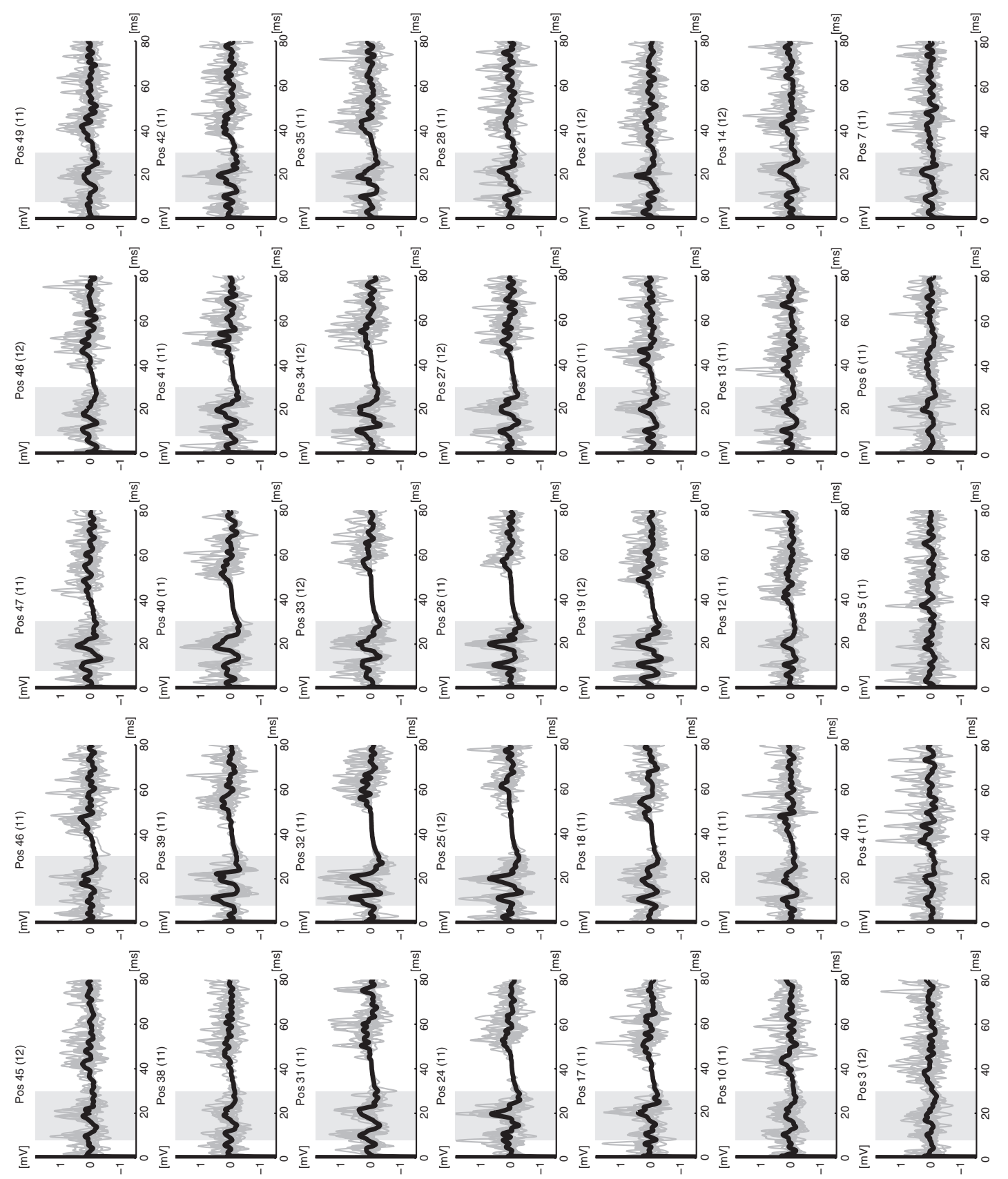

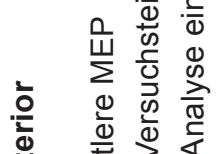

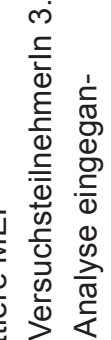

을 $\cong \frac{0}{\bar{c}}$

ल $\frac{0}{3}$ क

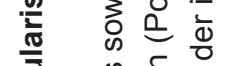

o d $\frac{5}{2}$

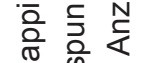

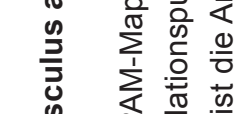

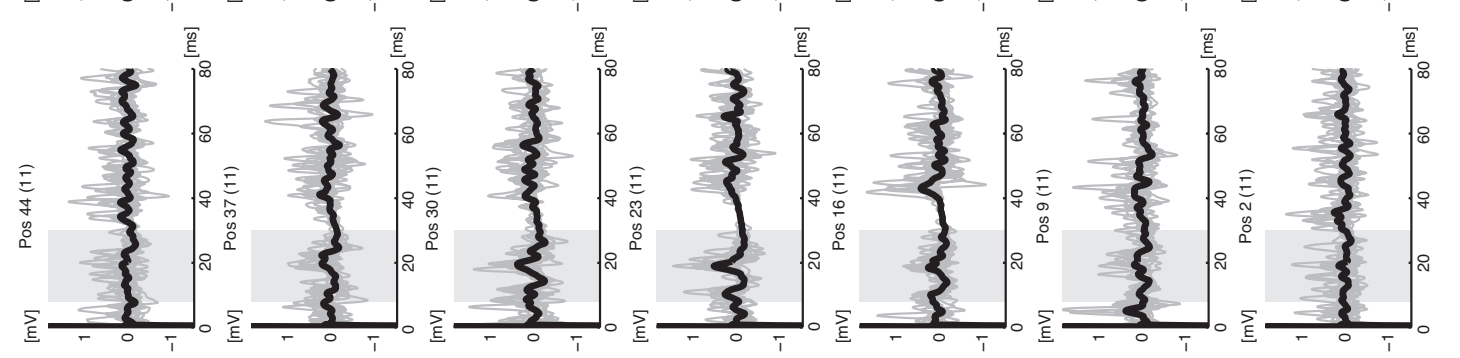

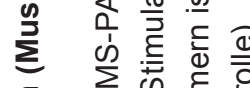

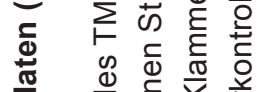

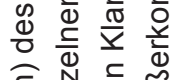

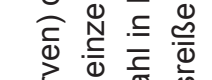

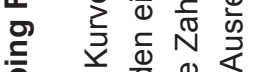

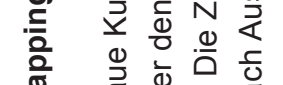
$\left\{\begin{array}{l}3 \\ 3\end{array}\right.$ 
Zur neuroanatomischen Lokalisation der kortikalen Repräsentationsareale wurden die individuellen Rasterpunkte, mittlere MEP-Amplituden und COGs auf die Gehirnoberfläche projiziert. Hierzu diente der Schnittpunkt zwischen Kortexoberfläche und Verlängerung der Vektoren der Stimulationsachse (durch den Mittelpunkt der TMS-Spule). Dieser Vektor befand sich dementsprechend rechtwinklig zur Tangentialebene über der Kopfoberfläche am jeweiligen Stimulationspunkt. Selbiger Vektor diente auch während der Experimente zur Einstellung der Spulenposition. Die Gruppenabbildung wurde erzeugt, indem die individuelle Hirnoberfläche der einzelnen ProbandInnen und die jeweiligen COGs mit der MNI152-T1-1mm-Gehirnschablone zusammengefügt wurden. Hierzu wurde FSL verwendet (BET, FNIRT and FLIRT). 


\section{ERGEBNISSE}

Bei allen ProbandInnen konnten MEP im PAM durch TMS innerhalb eines begrenzten Areals über der Kopfoberfläche, unabhängig von Geschlecht und individuellen Fähigkeiten, den Muskel vorzuaktivieren, und unter Anwendung von individualisierten Versuchsprotokollen hinsichtlich der Stimulationsintensität und Spulenlokalisation, ausgelöst werden.

\subsection{Motorisch evozierte Potenziale im Musculus auricularis posterior}

Bei 6 von 8 VersuchsteilnehmerInnen war eine Voraktivierung notwendig, um bei einer Stimulationsintensität von $\leq 75 \%$ der maximalen Stimulator Leistung (MSO) MEP im PAM auszulösen. In Abhängigkeit von den individuellen Fähigkeiten der ProbandInnen, den PAM willkürlich anzuspannen, zeigte sich eine hohe Variabilität der EMG-Amplituden während der Muskelaktivierung. So kam Versuchsteilnehmerln 2 auf eine EMG-Amplitude von 0,1 bis 0,51 mV (Mittelwert: 0,17 mV; Standardabweichung: 0,06 mV), während Versuchsteilnehmerln 4 eine Vorspannung zwischen 0,27 und 3,9 mV (Mittelwert: 1,7 mV; Standardabweichung: 0,51 $\mathrm{mV}$ ) erreichte.

Die MEP-Amplituden waren von der Stimulationsintensität abhängig (Abbildungen 4, 5 und 6). Bei 6 von den 8 ProbandInnen konnte das Verhältnis zwischen den relativen Werten derAmplituden und Stimulationsintensität in Form einer sigmoidalen Kurve dargestellt werden. Der Wendepunk befand sich hier zwischen 55\% und 79\% MSO (Abbildung 4). Bei den übrigen zwei Probandlnnen wurde bei maximaler Stimulationsintensität keine supra-maximale Stimulation erreicht, sodass sich kein Plateau einstellte, und auf Grund dessen eine Darstellung in Form einer sigmoidalen Kurve nicht möglich war. 


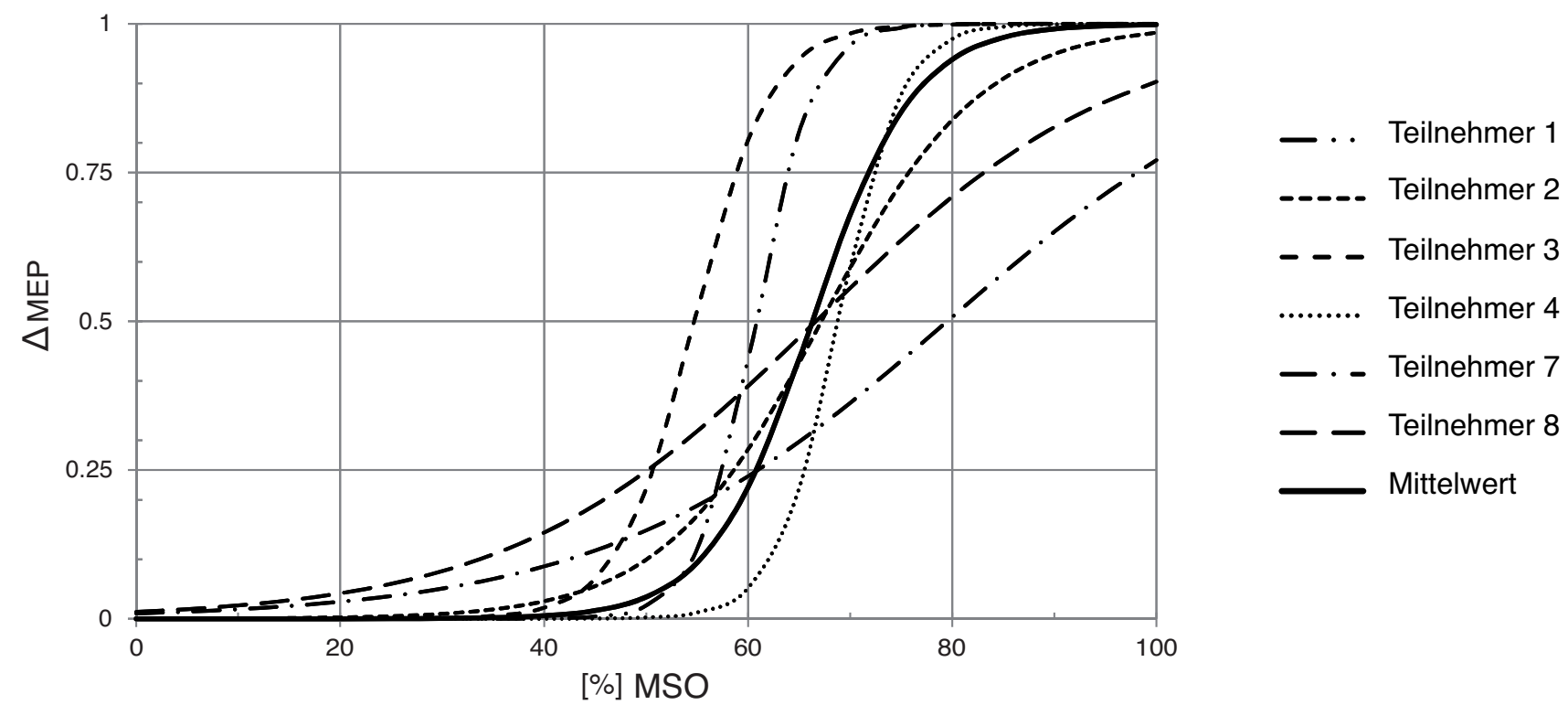

Abbildung 4: Amplituden der motorisch evozierten Potenziale im PAM in Abhängigkeit von der Stimulationsintensität

TMS-Rekrutierungskurven über den PAM-COGs. Die MEP wurden mit der folgenden Formel als Sigmoidfunktionen dargestellt: $f(x)=a m p n o s t i m+(\operatorname{ampmaxstim}-\operatorname{ampnostim}) /\left(1+\exp \left(m^{*}(W-x)\right)\right)$. ampnostim stellt die untere Ebene der EMG-Amplituden (Muskelvoraktivierung), ampmaxstim stellt die obere Ebene der EMGAmplituden, welches äquivalent zur maximalen MEP-Amplitude bei supramaximaler Stimulation ist. M ist die Steigung der Sigmoidfunktion am Wendepunkt W. Nach Anwendung einer Normalisierung der MEPAmplituden (ampnostim $=0$ und ampmaxstim $=1)$ resultierte die Funktion $\Delta M E P=1 /\left(1+\exp \left(m^{*}(W-x)\right)\right.$.

Auch die Dauer der MEP-Latenz war von der Stimulationsintensität abhängig. Am Wendepunkt betrug die mittlere MEP-Latenz 9,2 ms (Standardabweichung: 0,9 ms) und die peak-Latenz (Gipfel) 12,6 ms (Standardabweichung 1,1 ms). Bei zunehmender Stimulationsintensität zeigten sich eine Verkürzung der Latenz, sowie eine Verlängerung der silent period (Abbildungen 5 und 6). Die Latenz war zudem abhängig von der Spulenposition (Abbildung 3).

Die MEP-Form war variabel zwischen den Versuchsteilnehmerlnnen. Während die Mehrzahl der ProbandInnen (5 von 8) einen doppelgipfeligen Verlauf mit einem zweiten Gipfel von inkonsistenter Amplitude zeigte, war bei den restlichen Probandlnnen ein einzelner Gipfel zu verzeichnen (Abbildung 3, 5 und 7). 

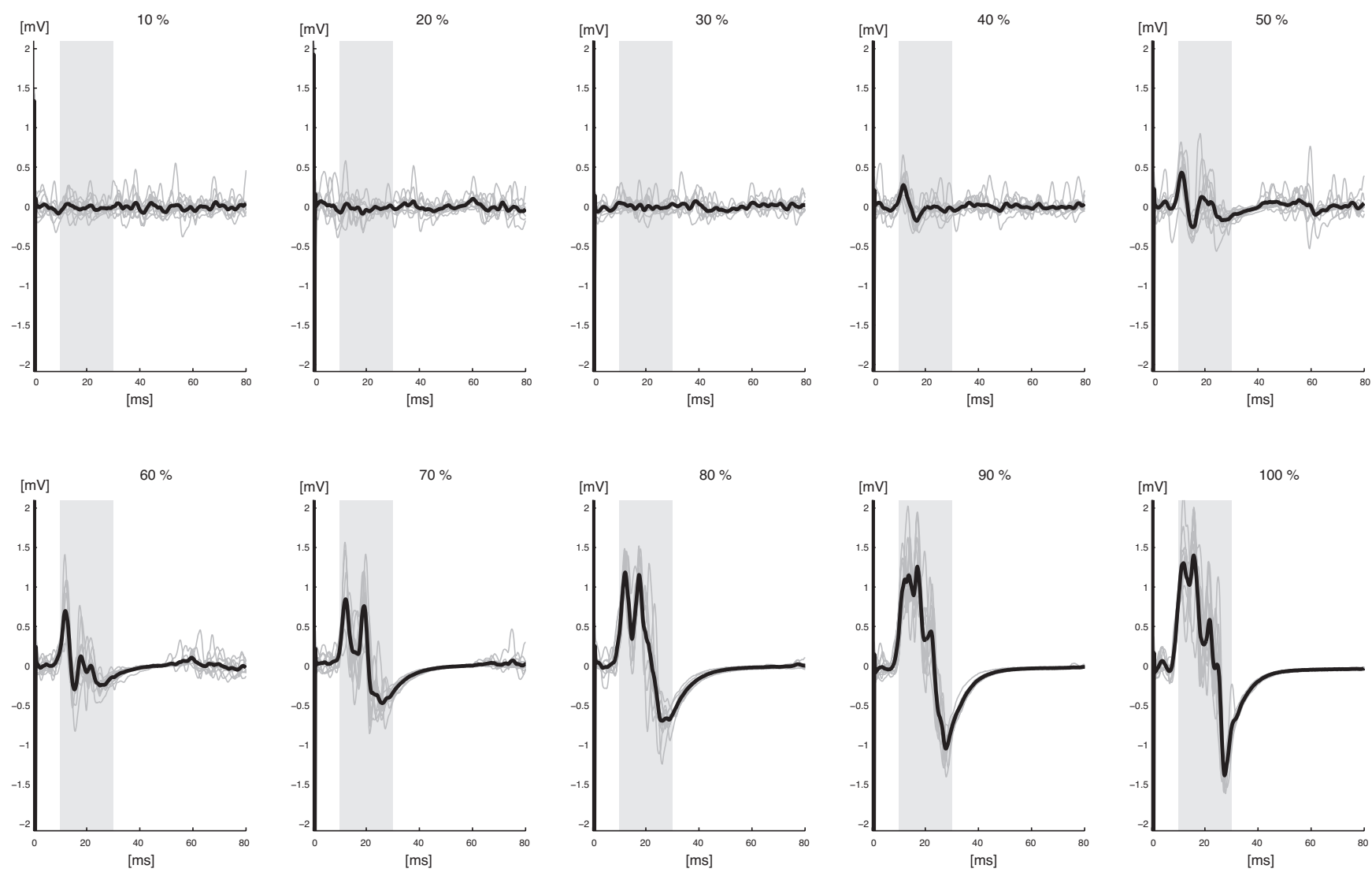

Abbildung 5: Motorisch evozierte Potenziale des Musculus auricularis posterior während Voraktivierung

Rekrutierungskurve, inklusive der Einzelmessungen (graue Kurven) und mittleren MEP (schwarze Kurven), über dem PAM-COG während Muskelvoraktivierung in Versuchsteilnehmerln 4. Es fällt eine positive Rekrutierung der MEP-Amplituden sowie eine Latenzverkürzung und Anstieg der Dauer der silent period in Abhängigkeit von der Stimulationsintensität auf.

Bei 3 der 8 ProbandInnen konnten MEP auch ohne Muskelvorspannung gemessen werden. Diese präsentierten einen solitären Gipfel und auch eine positive Rekrutierung (Abbildung 6). Ein Plateau wurde bei maximaler Stimulationsintensität nicht erreicht. Bei steigenden Stimulationsintensitäten zeigten sich hier eine Verkürzung der Latenzen sowie eine vermehrte Konsistenz der MEP in Bezug auf Form und Beginn (Abbildung 6). Im Kontrollexperiment wurde bei der für das TMS-Mapping-Experiment verwendeten Intensität in keinem der Versuchsteilnehmerlnnen ein PAMR nachgewiesen. 

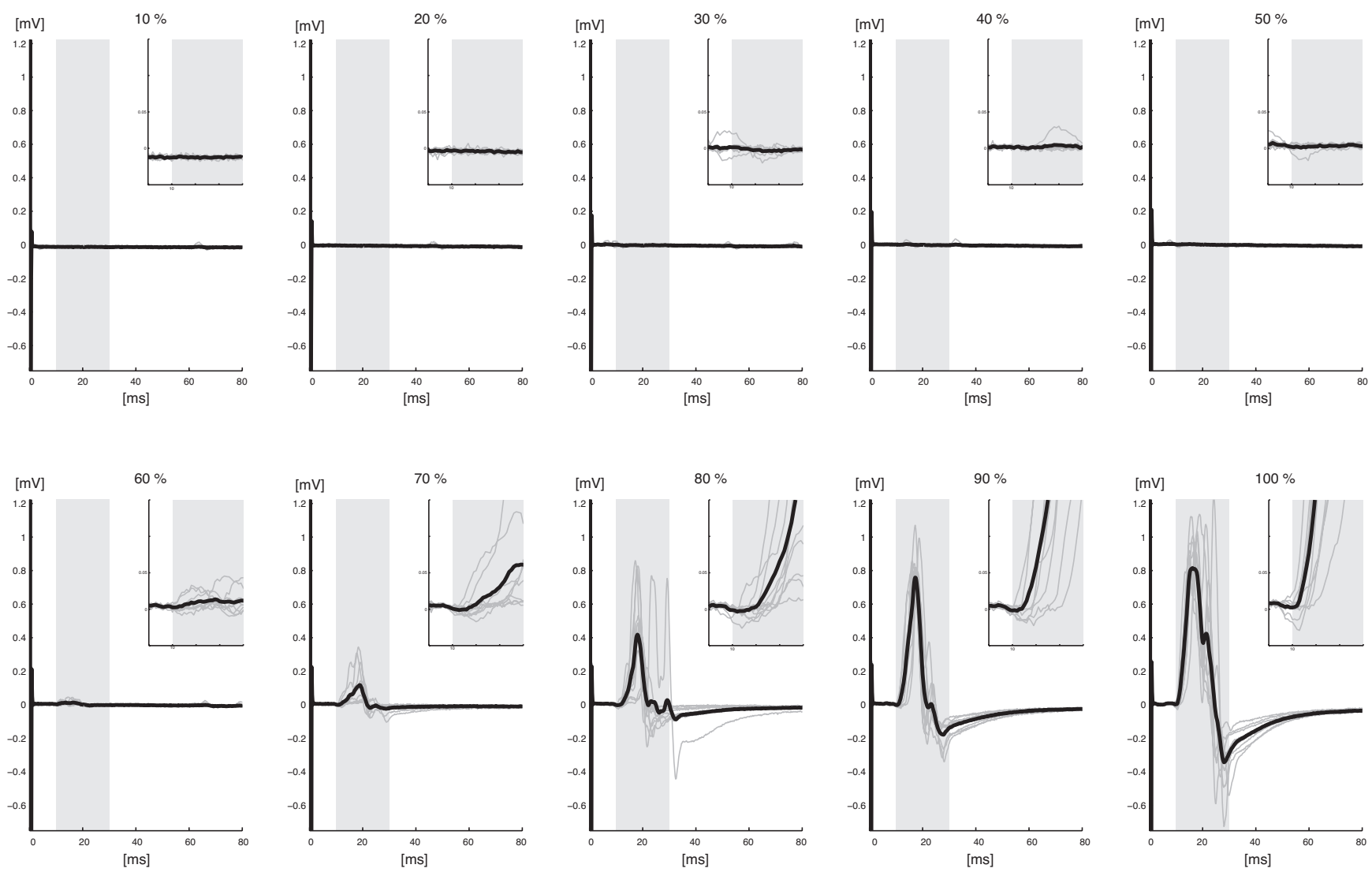

Abbildung 6: Motorisch evozierte Potenziale des Musculus auricularis posterior in Ruhe

Rekrutierungskurve, inklusive der Einzelmessungen (graue Kurven) und mittleren MEP (schwarze Kurven), über dem PAM-COG ohne Muskelvoraktivierung in Versuchsteilnehmerln 4 sowie vergrößerte Darstellung des jeweiligen MEP-Beginns. Es liegt eine positive Rekrutierung der MEP-Amplituden in Abhängigkeit der Stimulationsintensität vor. Supramaximale Stimulation wird nicht erreicht. Die Latenzen verkürzen mit steigender Stimulationsintensität. Bei hohen Stimulationsintensitäten fällt eine höhere Konsistenz der MEP in Bezug auf Form, Latenz und Amplitude auf. 

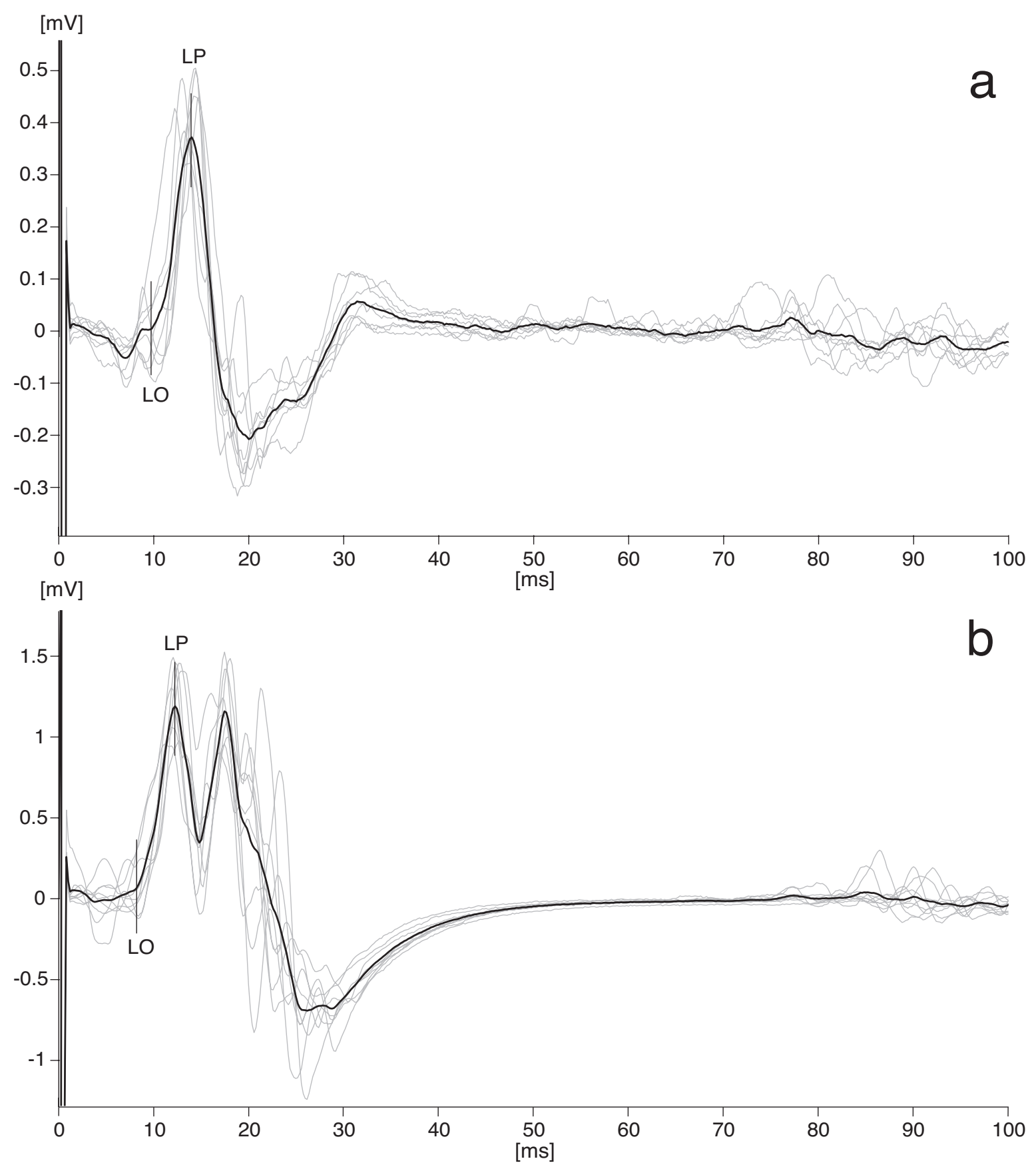

\section{Abbildung 7: Motorisch evozierte Potenziale am Center of Gravity}

Individuelle MEP (graue Kurven) und gemittelte MEP (schwarze Kurve) im voraktivierten PAM nach Stimulation über dem COG in Versuchsteilnehmerln 2 (a) und 7 (b), bei einer Stimulationsintensität, dem Wendepunkt der Rekrutierungskurve entsprechend (Versuchsteilnehmerln 2: 70\%; Versuchsteilnehmerln 7: $80 \% \mathrm{MSO}$ ). Bei $0 \mathrm{~ms}$ wurde der Stimulus appliziert. Die mittlere Latenz betrug 9,2 ms (Standardabweichung: 0,9 ms), die mittlere Peak-Latenz betrug 12,6 ms (Standardabweichung: 1,1 ms). 


\subsection{Mapping des Musculus auricularis posterior und Musculus inter-}

\section{osseus dorsalis I mittels transkranieller Magnetstimulation}

MEP im FDI wurden nach überschwelliger TMS über einigen Stimulationspunkten innerhalb des Rasters gemessen (Abbildungen 2 und 8). Das Areal, in dem die höchsten MEP-Amplituden ausgelöst wurden (mindestens 2/3 des Maximums) war nahezu kreisförmig mit einer Fläche von ca. 2,3 cm² (Standardabweichung: $\left.1,3 \mathrm{~cm}^{2}\right)$. Die COGs der jeweiligen Versuchsteilnehmerlnnen lagen innerhalb dieser Fläche.

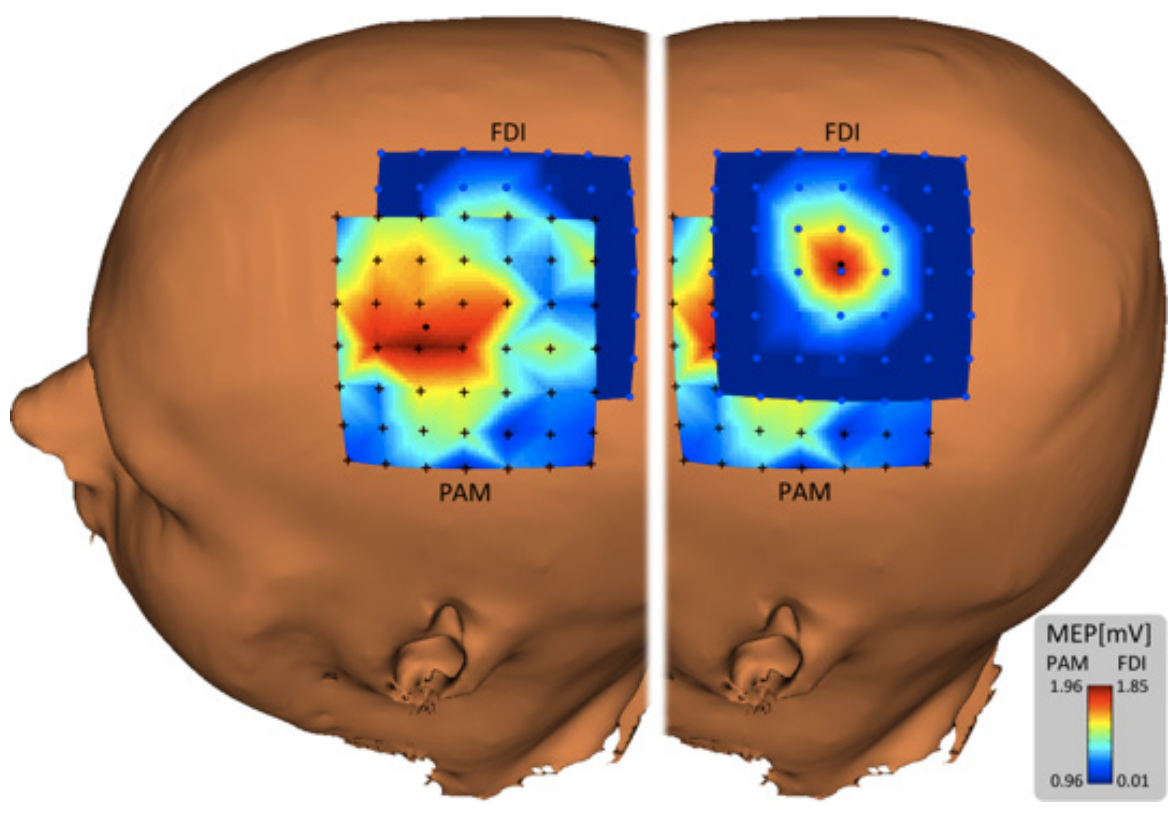

Abbildung 8: TMS-Mapping über der Kopfoberfläche

Projektion der individuellen TMS-Mapping-Ergebnisse (Amplituden), der COGs (schwarze Punkte) des PAM (links) und FDI (rechts) auf die Kopfoberfläche von Versuchsteilnehmerln 3. Die Stimulationspunkte sind als schwarze Kreuze (PAM) sowie blaue Punkte (FDI) dargestellt. Die im Vergleich zum FDI-Mapping hohe Grundlinienamplitude, welche das PAM-Areal umgibt, begründet sich aus der Muskelvorspannung. 
Durch die Stimulation von im Vergleich zu den FDI COGs einigen Zentimeter weiter lateral lokalisierten Zielpunkten wurden MEP im PAM ausgelöst (Abbildungen 3 und 8). Das Areal, in dem die MEP-Amplituden mindestens 2/3 des Maximums betrugen, war eher ovalförmig und hatte eine Größe von 7,5 cm² (Standardabweichung: 2,5 $\mathrm{cm}^{2}$ ). Die PAM COGs lagen bei allen Probandlnnen innerhalb dieser Fläche.

\subsection{Kortikale Repräsentation des Musculus auricularis posterior und Musculus interosseus dorsalis I}

In Bezug auf die Kortexoberfläche wurden die höchsten MEP-Amplituden im FDI nach Platzierung der TMS-Spule über dem sogenannten hand knob, welcher eine Windung des Gyrus praecentralis darstellt und sich etwa auf einer Ebene mit dem Gyrus frontalis medius befindet, ausgelöst (Abbildungen 9 und 10).

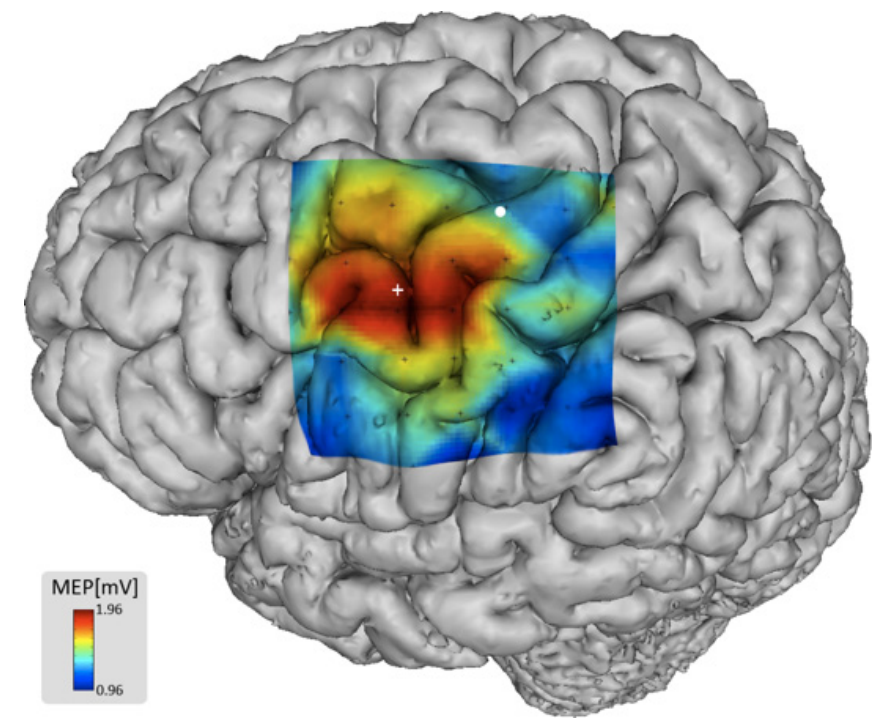

Abbildung 9: Kortexoberfläche und TMSMapping

Individuelle Ergebnisse des TMS-Mapping vom PAM von Versuchsteilnehmerln 3 als Projektion auf die Kortexoberfläche. Die Projektion der Stimulationspunkte (schwarze Kreuze), PAMCOG (weißes Kreuz), MEP-Amplituden (Farben) und FDI-COG (weißer Punkt) wurde ermittelt, indem der z-Vektor der TMS-Spule, welcher rechtwinklig zur Spulenoberfläche durch das Spulenzentrum verläuft, verlängert wurde und der Schnittpunkt mit der Kortexoberfläche markiert wurde. 
In 5 der 8 VersuchsteilnehmerInnen lagen die COGs im anterioren Abschnitt des Gyrus praecentralis nahe des Sulcus praecentralis. Bei den übrigen 3 ProbandInnen waren die COGs etwas weiter posterior, in der Mitte des Gyrus praecentralis, angeordnet (Abbildung 10). Die mittleren Koordinaten der COGs innerhalb des MNI152-Koordinatensystem waren [-36, -12 und 67]. Die Distanz zwischen den einzelnen COGs der Versuchsteilnehmerlnnen und dem mittleren COG betrug 5 mm (Standardabweichung: $1 \mathrm{~mm}$ ) (Abbildung 11).

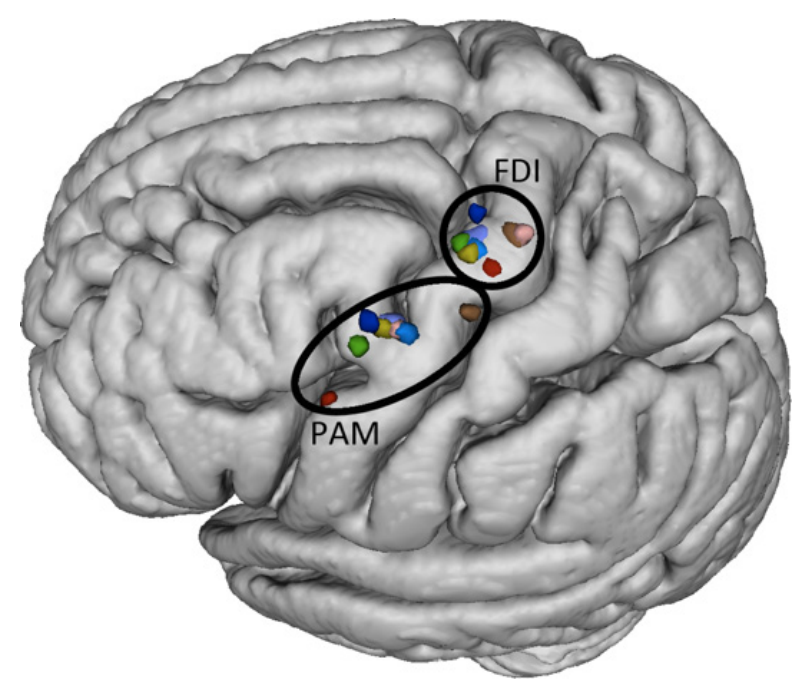

Abbildung 10: Kortikale Repräsentation des Musculus auricularis posterior und Musculus interosseus dorsalis

Lokalisation der individuellen COGs des PAM und FDI auf dem MNI152-T1-Template (standardisierte Gehirnschablone). Die Farben stehen für die unterschiedlichen Versuchsteilnehmerlnnen. Die mittlere Distanz zwischen den individuellen PAM- und FDI-COGs beträgt $23,8 \mathrm{~mm}$ (Standardabweichung: 5,1 mm).

Die höchsten MEP-Amplituden im PAM wurden nach Spulenpositionierung über im Vergleich zum FDI lateralen Anteilen des Gyrus praecentralis. In 6 von 8 ProbandInnen lagen die PAM-COGs etwa auf einer Ebene mit dem medialen Anteil des Gyrus frontalis inferior (Abbildungen 9 und 10). Bei den übrigen zwei Teilnehmern waren sie etwas weiter entfernt auf dem gleichen Gyrus lokalisiert, bei einem Versuchsteilnehmerln etwas weiter antero-lateral (in der Nähe des Sulcus praecentralis, auf einer Ebene mit dem lateralen Teil des Gyrus frontalis inferior) und bei einer/einem anderen Versuchsteilnehmerln etwas weiter postero-medial (in der Nähe des Sulcus centralis) (Abbildung 10). Die mittleren Koordinaten in- 
nerhalb des MNI152-Koordinatensystem waren [-50, 2 und 51]. Die mittlere Entfernung zwischen den einzelnen PAM COGs und dem mittleren PAM COG betrug $7 \mathrm{~mm}$ (Standardabweichung: $5 \mathrm{~mm}$ ) (Abbildung 11).

Die mittlere Distanz zwischen den individuellen COGs des PAM und FDI maß 23,8 mm (Standardabweichung: 5,1 mm). Die mittleren COGs waren 26,3 mm voneinander entfernt.

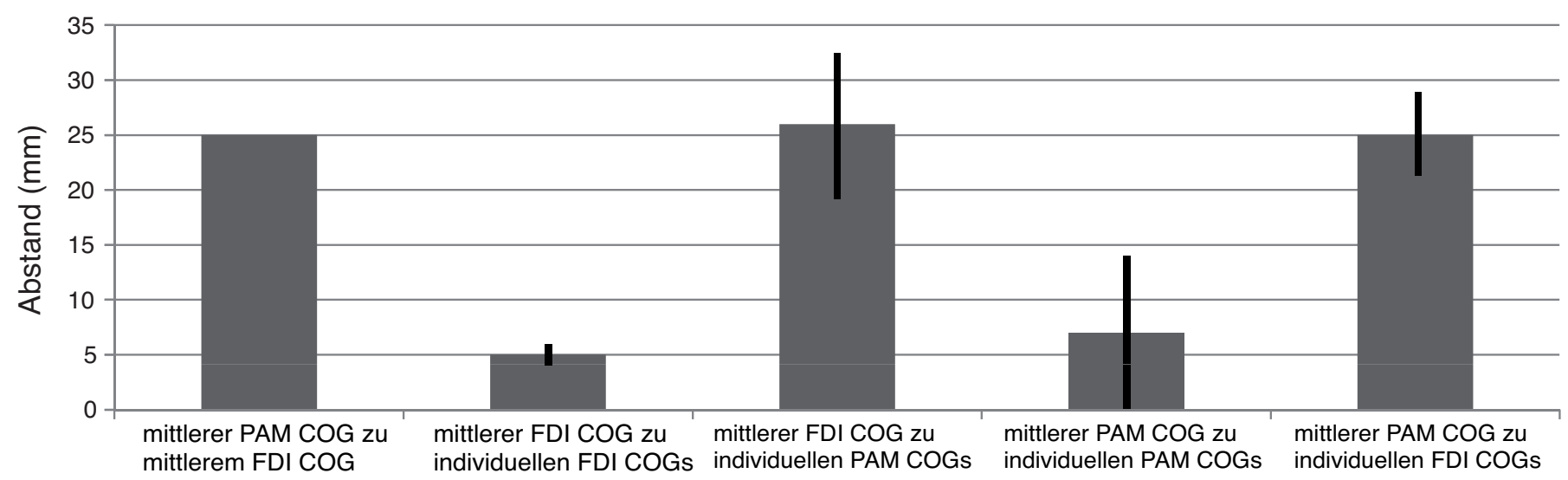

Abbildung 11: Entfernungen der kortikalen Repräsentationen der Musculi auriculares posteriores und Musculi interossei I

Entfernungen (in Millilmeter) zwischen den mittleren und individuellen COGs des PAM und FDI des TMSMappings. Die Fehlerbalken zeigen die Standardabweichung. 


\section{DISKUSSION}

In der vorliegenden Studie konnte die kortikale Repräsentation des PAM im Menschen mittels einer neuartigen neuronavigationsbasierten und robotergestützten TMS-Mapping-Technologie erstmalig identifiziert und lokalisiert werden. Das PAM-Repräsentationsareal stellte sich innerhalb des kontralateralen Gyrus praecentralis, lateral des Handareals, dar. Darüber hinaus zeigte die vorliegende Untersuchung, dass alle ProbandInnen in der Lage waren, den PAM mit Hilfe von visuellem und auditorischem Feedback nach Aufforderung zu aktivieren.

Im Folgenden werden Methodik und Ergebnisse der vorliegenden Untersuchung unter Einbeziehung bereits publizierter Daten in Bezug auf vergleichbare Themengebiete diskutiert.

\subsection{Motorisch evozierte Potenziale im Musculus auricularis posterior im Menschen}

Durch TMS über einem abgegrenzten Areal über der kontralateralen Hemisphäre wurden in allen VersuchsteilnehmerInnen MEP im PAM ausgelöst.

\subsubsection{Voraktivierung}

Bei 6 von 8 TeilnehmerInnen der Studie war bei Stimulationsintensitäten von $75 \%$ MSO oder niedriger eine Muskelvorspannung notwendig, um MEP im PAM auszulösen. Bei der Untersuchung von anderen fazialen Muskeln sind diesbezüglich die Ergebnisse heterogen. Während einige Forschergruppen keine Probleme bei 
Ableitungen unter Ruhebedingungen benannten (Benecke et al. 1988; Ghezzi et al. 1992), lieferten andere Studien erst unter Vorspannung positive Ergebnisse (Cruccu et al. 1990; Cruccu et al. 1997; Fischer et al. 2005).

Für diese widersprüchlichen Angaben kommen unterschiedliche Ursachen in Frage. Zum einen wurden abhängig von der jeweiligen Studie diverse mimische Muskeln untersucht, sodass eine Vergleichbarkeit nicht zwingend gegeben ist. Gleichwohl zeigten sich auch bei der Untersuchung derselben mimischen Muskeln diskrepante Ergebnisse bezüglich der Notwendigkeit einer Muskelvoraktivierung (Benecke et al. 1988; Cruccu et al. 1990; Kobayashi et al. 2001). Einen weiteren denkbaren ursächlichen Faktor für die bedeutend unterschiedlichen Ergebnisse stellt die Untersuchungsmethode bzw. die Durchführung der jeweiligen Studien dar. So unterschieden sich die Studienprotokolle in Bezug auf Stimulationsintensität und Spulentyp. Da die MEP-Amplituden eine positive Rekrutierung in Abhängigkeit von der Stimulationsintensität zeigen (Kiers 1993), ist ein Zusammenhang zwischen der Auslösbarkeit von MEP und Stimulationsintensität sowie Spulentyp wahrscheinlich. Auch in der vorliegenden Untersuchung zeigte sich eine positive Rekrutierung der PAM-MEP-Amplituden in Abhängigkeit von der Stimulationsintensität (Abbildungen 2 und 4). In 3 von 8 Versuchsteilnehmerlnnen konnten MEP im PAM außerdem ohne vorherige Muskelaktivierung ausgelöst werden (Abbildung 6). Ursächlich für diese interindividuell unterschiedlichen Ergebnisse könnten zum Beispiel unterschiedliche Stimulationsschwellen oder Divergenzen innerhalb der individuellen Neuroanatomie sein. Darüber hinaus wurden für unterschiedliche Gesichtsmuskeln in früheren Untersuchungen gezeigt, dass sich der optimale Rotationswinkel der TMS-Spule unterscheidet (Guggisberg et al. 2001; Dubach et al. 2004). Da es sich beim PAM um einen Muskel handelt, dessen korti- 
kale Repräsentatioin erstmalig untersucht wurde ist demzufolge die optimale Spulenausrichtung um MEP im PAM auszulösen unbekannt. Es ist dementsprechend denkbar, dass MEP im PAM bei optimaler Spulenrotation auch in Ruhebedingungen bei allen Probanden auslösbar wären. Hierzu wären weitere Untersuchungen am optimalen Stimulationsort unter Einbeziehung der Ausrichtung des individuellen primär motorischen Kortex notwendig.

\subsubsection{Latenzen}

Die hier gemessenen MEP-Latenzen im PAM (Mittelwert: 9,2 ms) rangieren im Vergleich mit vorangegangenen TMS-Studien anderer mimischer Muskeln im unteren Bereich. In der Literatur wurden bei der TMS anderer mimischen Muskeln Latenzen von 9,5 bis 13 ms beschrieben (Benecke et al. 1988, Ghezzi et al. 1992; Kobayashi et al. 2001; Fischer et al. 2005). Die relativ kurze Latenz der PAM MEP könnte zum einen in der Muskelvoraktivierung sowie der relativ hohen Stimulationsintensität begründet sein, wobei sich beide Faktoren neben einem Einfluss auf die Latenzdauer auch auf die Größe der MEP-Amplituden auswirken (Hess et al. 1986). Ein Einfluss von Stimulationsintensität und Vorspannung auf Latenzen und MEP-Amplituden konnte auch in der vorliegenden Untersuchung belegt werden (Abbildungen 4, 5 und 6). Eine weitere Ursache für die relativ kurzen Latenzen könnte mit dem relativ kurzen peripheren Verlauf des Astes des N. facialis, der den PAM innerviert, in Verbindung stehen (Henle 1871).

\subsubsection{Blinkreflex}

In der Literatur wurde immer wieder eine mögliche Verunreinigung der gemes- 
senen fazialen MEP durch den Blinkreflex, welcher nach einer vergleichbaren Latenz auftritt und dessen Morphologie jener der MEP ähnelt, diskutiert (Cocito und Cassano 1993; Sohn et al. 2004). Den afferenten Schenkel des Blinkreflexes bilden Schmerzfasern des N. trigeminus, der efferente Schenkel setzte sich aus N. facialis und M. orbicularis oculi zusammen. Da durch die TMS auch afferente Trigeminus-Schmerzfasern direkt stimuliert werden, kann durch TMS der Blinkreflex, d. h. eine reflektorische (unwillkürliche) Kontraktion des M. orbicularis oculi ausgelöst werden. Eine Aufzeichnung von entfernten Muskelpotenzialen (z.B. des M. orbicularis oculi im Rahmen des Blinkreflexes) sowie eine direkte Fortleitung der Muskelaktivität in benachbarte Muskulatur könnte daraufhin MEP-Aufzeichnungen anderer mimischer Muskeln beeinflussen (Bischoff et al. 1993).

Auch in der vorliegenden Studie ist eine solche Kontaminierung der gemessenen MEP denkbar. Dagegen spricht jedoch die direkte Platzierung der Drahtelektroden innerhalb des PAM sowie seine relativ isolierte Lage hinter der Ohrmuschel, die eine Fortleitung von aus dem M. orbicularis oculi ausgehenden Muskelpotenzialen in den PAM (über andere mimische Muskeln) sowie eine Aufzeichnung von anderen entfernten Muskelpotenzialen unwahrscheinlich machen (English und Weeks 1989). Ein weiteres Argument gegen eine mögliche Aufzeichnung des Blinkreflexes anstelle von MEP und für den kortikalen Ursprung der Potenziale ist das klar definierte Areal, durch dessen Stimulation MEP ausgelöst wurden. Der Blinkreflex hätte dementsprechend nach TMS über allen Rasterpunkten gleichermaßen hervorgerufen werden müssen, da das komplette Raster ein durch den N. trigeminus versorgtes Gebiet bedeckte. Auch die silent period spricht für einen kortikalen Ursprung der gemessenen Potenziale (Wilson et al. 1993). 
Weiterhin spricht die Identifizierung eines vergleichbaren kortikalen Repräsentationsareals mittels funktioneller Bildgebung (s. u.) für einen kortikalen Ursprung der MEP und gegen eine Aufzeichnung des Blinkreflexes.

\subsubsection{Musculus-auricularis-posterior-Reflex}

Ein weiterer möglicher Störfaktor von MEP im PAM ist der Musculus-auricularis-posterior-Reflex (PAMR), eine reflektorische Kontraktion des PAM, die durch bestimmte Geräusche hervorgerufen wird. Um auszuschließen, dass der PAMR bei den VersuchsteilnehmerInnen durch das Geräusch des TMS-Stimulus getriggert wird, wurde ein Kontrollexperiment durchgeführt. Ähnlich wie beim bereits beschriebenen Blinkreflex gilt auch bei diesem Reflex, dass ein klar definiertes kortikales Repräsentationsareal sowie die silent period für einen kortikalen und gegen einen reflektorischen Ursprung der gemessenen MEP sprechen (Wilson et al. 1993).

\subsection{Kortikales Repräsentationsareal des Musculus auricularis posterior und Musculus interosseus dorsalis I}

MEP wurden sowohl im PAM als auch im FDI nach Stimulation von Zielpunkten innerhalb einer abgegrenzten Fläche über der Kopfoberfläche ausgelöst. Die Fläche, durch deren Stimulation MEP im FDI ausgelöst wurde, war kleiner und klarer begrenzt als jene des PAM (Abbildung 8). Auch die Streuung der COGs, d. h. die Entfernung zwischen den individuellen und dem mittleren COGs, war größer für den PAM im Vergleich zum FDI (Abbildungen 10 und 11). Dieses könnte möglicherweise in einer höheren neuroanatomischen Variabilität zwischen den Versuchs- 
teilnehmerlnnen in Bezug auf die kortikale PAM-Repräsentation begründet sein. Als weiterer Grund für diese Unterschiede kommen die verschieden aufgebauten Mapping-Protokolle, die für die Untersuchung beider Muskeln verwendet wurden, in Betracht. Da sich die PAM-MEP bei niedrigeren Stimulationsintensitäten nicht eindeutig von den Muskelpotenzialen im Rahmen der Voraktivierung abgrenzen ließen, wurde für das PAM-Mapping eine im Vergleich zum FDI-Mapping relativ höhere Stimulationsintensität verwendet. Als Folge einer höheren Stimulationsintensität sinkt jedoch das räumliche Auflösungsvermögen der TMS. Dies bedeutet, dass eine Stimulation einer hohen Anzahl von Rasterpunkten eine überschwellige Erregung im selben Neuron auslösen kann (Krings et al. 1998). Als Folge einer hohen Stimulationsintensität ist aus diesem Grund, neben der Auslösung von höheren MEP-Amplituden, eine verminderte räumliche Abgrenzbarkeit des Repräsentationsareals zu benennen (Krings et al. 1998), sodass die Ausdehnung des kortikalen Repräsentationsareals des PAM weitläufiger als jene des FDI erscheint und eine mögliche Ursache für die im Vergleich zu den FDI COGs größere Streuung der PAM COGs darstellen könnte.

Im Vergleich zu vergangenen TMS-Mapping-Experimenten waren die ermittelten Repräsentationsareale jedoch klar abgrenzbar und die Fläche vergleichbar gering (Wassermann et al. 1992; Reilly und Mercier 2008). Eine mögliche Ursache dafür stellen die hohe Anzahl von TMS-Pulsen über jedem Rasterpunkt und die Verwendung von mittleren MEP-Amplituden für die Datenanalyse dar. So wurde der Einfluss von Ausreißerpotenzialen und der hohen natürlichen Variabilität der MEP-Amplituden (Kiers et al. 1993; Wassermann et al. 2002; Jung et al. 2010) auf die Daten vermindert. Weitere mögliche Gründe sind die randomisierte Stimulationsabfolge sowie die Verwendung der hochpräzisen und roboterkontrollierten 
Mapping-Technologie zur Spulenpositionierung (siehe unten).

\subsection{Lokalisation der kortikalen Repräsentation des Musculus auricularis posterior und Musculus interosseus dorsalis I}

Auf die Kortexoberfläche projiziert, befanden sich die COGs des PAM und FDI signifikant voneinander entfernt auf dem Gyrus praecentralis, wobei die COGs des FDI alle im Bereich des sogenannten hand knob und die PAM COGs einige Zentimeter weiter lateral lokalisiert waren. Eine vergleichbare Neuroanatomie konnte mittels TMS-Mapping und funktioneller Bildgebung bereits für andere mimische Muskeln und Handmuskeln dargestellt werden (Wassermann et al. 1994; Lotze et al. 2000; Paradiso et al. 2005; Meier et al. 2008; Weiss et al. 2012). Es ist daher anzunehmen, dass sich durch TMS-Mapping kein Unterschied der kortikalen Repräsentation zwischen aurikulären Muskeln und anderen mimischen Muskeln darstellen lässt (ggf. überlappende Repräsentationsareale). Eine überlappende kortikale Repräsentation von in der Peripherie angrenzenden bzw. nahe beieinander lokalisierten Muskeln wurde bereits für mimische und Extremitäten-Muskulatur mit unterschiedlichen (bildgebenden) Verfahren (u. a. TMS und fMRT) gezeigt (Wilson et al. 1993; Meier et al. 2008, Weiss et al. 2012).

\subsection{Funktionelle Bildgebung während Aktivierung des Musculus auri- cularis posterior und Musculus interosseus dorsalis I}

Eine weitere Methode zur Erforschung von (kortikalen) Repräsentationsarealen unterschiedlicher Bewegungen (Muskeln) stellt die funktionelle Magnetresonanztomographie dar, welche das Maß der Blutoxygenierung (BOLD) erfasst. Die Pla- 
nung und Durchführung von Willkürbewegungen führt zu verstärkter neuronaler Aktivierung, welche wiederum eine verstärkte Perfusion von oxygeniertem Blut zur Folge hat, die mittels fMRT lokalisiert und mit Ruhebedingungen verglichen werden kann.

Nach Während einer nach hinten gerichteten Bewegung der Ohrmuscheln und einer nach medial gerichteten Bewegung der Zeigefinger zeigte sich im fMRT ein erhöhtes BOLD-Signal innerhalb von M1, sensorischem Kortex, SMA, Inselregion und Basalganglien. Die Zeigefingerbewegung resultierte darüber hinaus in einem verstärkten BOLD-Signal innerhalb des sekundär motorischen Kortex. Die stärkste Aktivierung zeigte sich für beide Bewegungen innerhalb von M1. Die COGs für die Fingerbewegungen waren sulcal innerhalb des hand knob und der Ohrbewegung einige Zentimeter weiter lateral lokalisiert (Veröffentlichung in Arbeit). Diese Ergebnisse stimmen mit den Ergebnissen vorangehender fMRT-Untersuchungen während Aktivierung von Handmuskeln und mimischen Muskeln überein (Lotze et al. 2000; Meier et al. 2008; Weiss et al. 2012).

Insgesamt war die Aktivierung während der Fingerbewegung deutlich stärker als während der Ohrbewegung. Dieses Ergebnis entspricht den Ergebnissen anderer fMRT-Studien, in denen ein stärkeres BOLD-Signal während Fingerbewegungen im Vergleich zu anderen Bewegungen der Extremitäten oder Gesichtsmuskulatur gezeigt wurde (Lotze et al. 2000; Meier et al. 2008). 


\subsection{Vergleich der Mapping-Ergebnisse der transkraniellen Magnetstimu- lation mit der funktionellen Magnetresonanztomographie}

Der Vergleich fMRT- und TMS-Mapping der kortikalen Repräsentation der aurikulären Muskulatur zeigt große Übereinstimmungen im Bereich der ermittelten Repräsentationsareale beider Muskeln bzw. Bewegungen. Obwohl die TMS- und fMRT-Ergebnisse ein hohes Maß an Korrelation in Bezug auf die Anordnung zwischen Ohr- und Handmuskeln zueinander aufweisen, ist die Lokalisation der COGs nicht identisch. Bei allen Probandlnnen und unabhängig vom untersuchten Muskel befanden sich die TMS-COGs etwas weiter anterior im Vergleich zu den fMRT-COGs (Veröffentlichung in Arbeit). Dieses Phänomen hat sich bereits in anderen Studien, die TMS und fMRT verglichen, dargestellt (Terao et al. 1998; Herwig et al. 2002; Lotze et al. 2003). Während die ursächliche Bestimmung dieses Unterschieds unbekannt ist, sind die möglichen Einflussfaktoren vielfältig und in den Unterschieden beider Methoden begründet: Die fMRT misst einen erhöhten Blutsauerstoffgehalt, der sich während neuronaler Aktivierung einstellt. Im Gegensatz dazu werden bei der TMS Nervenzellen, insbesondere deren Axone, stimuliert. Im Falle der Stimulation von kortikalen motorischen Nervenzellen wird der Reiz weitergeleitet und kann im Zielmuskel als MEP abgeleitet werden. Auf Grund physikalischer Eigenschaften sind Axone, die zunächst parallel zum elektrischen Feld verlaufen und sich später biegen besonders vulnerabel für eine solche Stimulation (Amassian et al. 1992; Maccabee et al. 1993; Kobayashi und Pascual-Leone 2003). Dementsprechend ist anzunehmen, dass die Lokalisation eines durch TMS aktivierten Axons nicht unbedingt jener der erhöhten Blutoxygenierung entspricht, die beim fMRT gemessen wird und insbesondere durch den verstärkten Metabolismus innerhalb der Nervenzellkörper ausgelöst wird. Ein wei- 
terer Grund für den Unterschied zwischen TMS und fMRT ist durch das elektrische Feld, welches für die durch TMS ausgelöste Stimulation der Nervenzellen verantwortlich ist, begründet. Dieses elektrische Feld ist nicht punktgenau unter dem Spulenmittelpunkt verortet sondern breitet sich abhängig von der Richtung des Stromflusses in verschiedene Richtungen in unterschiedlichem Maß aus (Maccabee et al. 1993; Kobayashi und Pascual-Leone 2003). Es ist also denkbar, dass die tatsächliche neuronale Aktivierung durch TMS nicht unmittelbar unterhalb des Spulenmittelpunkts lokalisiert ist und infolgedessen eine Verschiebung zwischen tatsächlichem und angenommenen Stimulationsort stattfindet. Ein weiterer Faktor, der beim Vergleich von TMS- und fMRT-Daten berücksichtigt werden muss, ist, dass der genaue Wirkort von TMS nicht genau bekannt ist. Es wird jedoch angenommen, dass die neuronale Aktivierung, vergleichbar mit jener die im fMRT nachgewiesen wird, am ehesten in der Tiefe des Sulcus innerhalb des primär motorischen Kortex stattfindet (Fox et al. 2004). Da die TMS-Daten jedoch zur Veranschaulichung meist auf die Oberfläche projiziert werden, repräsentieren sie nicht die genaue Lokalisation des Repräsentationsareals (in der Tiefe des Sulcus). Es ist daher anzunehmen, dass die Darstellungsmethode den Methodenvergleich beeinflusst bzw. verzerrt. Als weitere Ursache für den Unterschied kommt die fehlende Muskelspezifität des fMRT in Frage. Die während der fMRT-Untersuchung durchgeführten Bewegungen beinhalteten neben einer starken Aktivierung der untersuchten Muskeln auch die Aktivierung anderer Muskulatur, die an den ausgeführten Bewegungen beteiligt ist. Im Falle eines Nicht-identischen Repräsentationsareal der an der Bewegung beteiligten Muskulatur könnte aus diesem Grund ein fMRT-Ergebnis beeinflusst werden. 


\subsection{Robotergestützte und neuronavigationsbasierte transkranielle Magnetstimulation}

In der vorliegenden Studie wurde ein robotergestütztes und neuronavigationsbasiertes TMS-Mapping-Protokoll verwendet. Bezüglich der Reliabilität der MappingErgebnisse konnte in vergangenen Untersuchungen bereits eine Überlegenheit neuronavigierter TMS im Vergleich zur klassischen TMS nachgewiesen werden (Gugino et al. 2001; Denslow et al. 2005; Sparing et al. 2008; Julkunen et al. 2009).

Die neuronavigierte TMS wurde in der vorliegenden Studie durch die Anwendung einer hochpräzisen robotergestützten Spulenpositionierung weiter verbessert. Im Hinblick auf die 6 Freiheitsgrade im Raum wurde die Spulenposition durch den Roboterarm permanent kontrolliert sowie jeglicher Einfluss des Untersuchers auf die Ergebnisse minimiert. Parallel dazu wurde die Position des ProbandlnnenKopfes über ein Infrarot-Kamerasystem mit der Spulenposition abgeglichen und Kopfbewegungen dadurch ausgeglichen. Diese Methode gewährleistete die Einhaltung eines konstanten Abstandes zwischen Spule und Kopfoberfläche sowie eines konstanten Rotationswinkels. So wurden Verschiebungen des durch TMS ausgelösten elektrischen Feldes und damit ein Einfluss auf MEP vermieden (Mills et al. 1992; Richter et al. 2013a; Richter et al. 2013b) und gleiche Messbedingungen für jeden einzelnen TMS-Puls sichergestellt.

Einer früheren Version dieses robotergestützten und neuronavigationsbasierten TMS-Systems wurde bereits in zurückliegenden Untersuchungen ein hohes Maß an Reliabilität während der Sitzung sowie zwischen verschiedenen Sitzungen hinsichtlich der stimulierten Rasterpunkte zugesprochen (Kantelhardt et al. 2010). In der vorliegenden Studie wurde das o. g. TMS-System in verschiedener Hinsicht weiterentwickelt. So wurde während der TMS-Sitzungen eine Gütekontrolle der 
Messungen durchgeführt. Im Falle des Nicht-Erreichens der nötigen Vorspannung während einer PAM-Messung bzw. einer fälschlicherweise durchgeführten Präaktivierung des FDI wurden jene Messungen automatisch wiederholt. Wegen der hohen Reliabilität in Bezug auf die Wiederauffindbarkeit von Stimulationspunkten konnten die verschiedenen Mapping-Experimente auf mehrere Sitzungen verteilt werden. Darüber hinaus wurde eine im Vergleich zu früheren Untersuchungen, in denen meist zwischen 3 und 6 Stimuli pro Punkt abgegeben wurden (Lotze et al. 2003; Sparing et al. 2008), relativ hohe Anzahl von Stimuli pro Punkt verwendet. Demenstprechend wurde der Einfluss der natürlichen Variabilität der MEP-Amplituden auf die Mapping Ergebnisse vermindert (Kiers et al. 1993; Wassermann et al. 2002).

Da Unterschiede des Aufmerksamkeits- und Wachheitsniveaus der ProbandInnen während der TMS-Sitzungen erheblichen Einfluss auf MEP-Amplituden nehmen (Ziemann et al. 1996) wurde erstmalig eine vollständig randomisierte Stimulationsreihenfolge verwendet. Aufmerksamkeitsunterschiede wurden so über das Raster ausgeglichen und sollten das Mapping-Ergebnis nicht beeinflusst haben. Eine TMS-Mapping-Sitzung dauerte ca. 100-110 Minuten, inklusive einer Vorbereitungszeit von ca. 20 Minuten. Die Dauer der Mapping-Sitzung rangiert damit im mittleren bis oberen Bereich verglichen mit früheren TMS-Mapping Untersuchungen (Kleim et al. 2007; Guerra et al. 2014). 


\section{SCHLUSSFOLGERUNG UND AUSBLICK}

Mittels einer neuartigen robotergestützten und neuronavigationsbasierten TMSMapping-Technologie konnte eine kortikale Repräsentation der aurikulären Muskulatur im Menschen erstmalig dargestellt und lokalisiert werden. Dieses Repräsentationsareal befindet sich innerhalb des primär-motorischen Kortex lateral des Handareals. Darüber hinaus konnte gezeigt werden, dass alle Probandlnnen die Fähigkeit besitzen, den PAM mit Hilfe von auditorischem und visuellem EMGFeedback zu aktivieren. Diese Ergebnisse sind in vielerlei Hinsicht von besonderem Interesse.

Zum einen wurde in tract-tracing-Experimenten im Rhesusaffen festgestellt, dass sich die kortikale Repräsentation der aurikulären Muskulatur vor allem in kontralateralen supplementär motorischen- und cingulären Kortexarealen und nur marginal innerhalb des primär motorischen Kortex befindet (Jenny und Saper 1987, Morecraft et al. 2001). Die in der vorliegenden Studie nachgewiesene kortikale Repräsentation der humanen aurikulären Muskeln, welche vorzugsweise innerhalb des primär motorischen Kortex lokalisiert ist, zeigt demzufolge bis dato nicht bekannte neuroanatomische Unterschiede zwischen Menschen und Rhesusaffen auf.

In der Literatur wurde dem PAM eine spezifische Funktion im Rahmen der Mimik oder auch die Möglichkeit einer eigenständigen willkürlichen Bewegung oftmals abgesprochen (Henle 1858; Serra et al. 1986). Es wurde angenommen, seine einzige Funktion läge in einer reflektorischen Aktivierung bzw. der daraus resultierenden Positionierung der Ohrmuschel im Rahmen einer unwillkürlichen Rich- 
tungsortung von Geräuschen. Das hier identifizierte kortikale Repräsentationsareal des PAM innerhalb des primär motorischen Kortex und die Tatsache, dass alle Probandlnnen den PAM auf Aufforderung aktivieren konnten, widerspricht jedoch dieser Darstellung und weist auf eine darüber hinausgehende, weiterentwickelte Funktion der aurikulären Muskeln im Menschen hin. Hierbei käme u. a. eine Mitbeteiligung innerhalb von Gesichtsausdrücken in Frage. Eine solche Funktion könnte z. B. im Rahmen von weiterführenden EMG-Untersuchungen an den aurikulären Muskeln sowie anderen mimischen Muskeln ermittelt werden. Auch die Möglichkeit einer möglichen muskelspezifischen Aktivierung der aurikulären Muskulatur, d. h. ohne zeitgleiche Aktivierung anderer mimischer Muskeln (ggf. auch der einzelnen aurikulären Muskeln unabhängig voneinander), könnte im Anschluss an die geschilderten Ergebnisse im Rahmen weiterer Untersuchungen erforscht werden.

Neben den neuroanatomischen Erkenntnissen zeigte die vorliegende Untersuchung auch, dass die Methodik von TMS-Mapping durchaus Verbesserungsund Weiterentwicklungspotenzial birgt. Mittels der hier verwendeten neuartigen robotergestützten Technologie zur Spulenpositionierung konnte erstmalig eine vollständig randomisierte Stimulationsabfolge erfolgen und so der Einfluss von Fluktuationen des Wachheitszustandes/der Probandln über das Experiment ausgeglichen werden. Darüber hinaus wurde durch die robotergestützte Spulenpositionierung der Einfluss des Untersuchers auf die Ergebnisse minimiert. Auf Grund der automatischen Kontrolle (und ggf. Wiederholung) der MEP auf Vorspannung mussten die Daten nicht offline auf ihre Güte kontrolliert werden, sondern wurden allesamt in die Analyze einbezogen.

Nichtsdestotrotz kann TMS-Mapping weiter optimiert werden. So ist es denkbar, 
dass auf Grund der hohen Präzision des Roboters, weniger TMS-Pulse pro Stimulationspunkt zu reliablen Ergebnissen führen könnten. Hierzu würde sich eine Varianz- und Reliabilitätsnalyse in Abhängigkeit zur Anzahl der abgegebenen Pulse anbieten. Eine weitere mögliche Weiterentwicklung der Mapping-Technologie besteht in der Entwicklung eines vollständig automatischen Experimentenprotokoll, welches neben des eigentlichen Mappings auch die Suche nach geeingneten Stimulationspunkten beinhaltet. Neben einer kürzeren Experimentendauer durch Fokus der Untersuchung auf das kortikale Repräsentationsareal, würde dadurch jeglicher Einfluss des Untersuchers vermieden werden. Des weiteren bietet die hochpräzise Spulenpositionierung die Möglichkeit, die Auflösung von TMS-Mapping, z. B. durch die Verwendung eines kleineren Abstandes zwischen den Stimulationspunkten, erheblich zu erhöhen. Durch solch eine Weiterentwicklung könnten zahlreiche neue Erkenntnisse bezüglich der genauen Wirkweise von TMS bzw. des genauen Wirkortes erlangt werden. 


\section{PERSPEKTIVEN}

Die Aktivierung der aurikulären Muskulatur, bzw. die Möglichkeit des Erlernens einer (muskelspezifischen) Aktivierung, könnte innerhalb von Mensch-MaschineSchnittstellen wie der Steuerung von Rehabilitationsmitteln - z. B. von Rollstühlen oder Prothesen - Verwendung finden. Solch ein EMG-basiertes Steuerungssystem könnte sogar bei Patienten mit hohen Querschnittssyndromen Anwendung finden, da hier vor dem Hintergrund der bisherigen Ergebnisse mit keiner Mitbeteiligung des $\mathrm{N}$. facialis zu rechnen ist. Im Vergleich zu bereits existierenden Steuerungssystemen, die insbesondere auf Augen- oder Zungenbewegungen beruhen, würde die Nutzung von Muskelpotenzialen aus der aurikulären Muskulatur zu weniger Einschränkungen in alltäglichen Tätigkeiten, wie z. B. sprechen oder essen führen. Für die betroffenen Patienten hätte ein solches Steuerungssystem dementsprechend einen Zugewinn an Lebensqualität und Autonomie zur Folge. 


\section{LITERATURVERZEICHNIS}

Amassian VE, Stewart M, Quirk GJ, Rosenthal JL (1987): Physiological basis of motor effects of a transient stimulus to cerebral cortex. Neurosurgery $\underline{20}$, $74-93$

Amassian VE, Eberle L, Maccabee PJ, Cracco RQ (1992): Modelling magnetic coil excitation of human cerebral cortex with a peripheral nerve immersed in a brain-shaped volume conductor: the significance of fiber bending in excitation. Electroencephalogr Clin Neurophysiol $\underline{85}, 291-301$

Awiszus F (2003): TMS and threshold hunting. Suppl Clin Neurophysiol $\underline{56}, 13-23$

Barker AT, Jalinous R, Freeston IL (1985): Non-invasive magnetic stimulation of human motor cortex. Lancet 1985, I, 1106-7

Bartholow R (1874): Experimental investigations into functions of the human brain. Am J Med Sci $\underline{67}, 305-313$

Benecke R, Meyer BU, Schonle P, Conrad B (1988): Transcranial magnetic stimulation of the human brain: responses in muscles supplied by cranial nerves. Exp Brain Res $\underline{71}$, 623-32

Benning SD (2011): Postauricular and superior auricular reflex modulation during emotional pictures and sounds. Psychophysiology $\underline{48}$, 410-4

Berzin F, Fortinguerra CR (1993): EMG study of the anterior, superior and posterior auricular muscles in man. Ann Anat $\underline{175}, 195-7$

Bischoff C, Liscic R, Meyer BU, Machetanz J, Conrad B (1993): Magnetically elicited blink reflex: an alternative to conventional electrical stimulation. Electromyogr Clin Neurophysiol $\underline{33}, 265-9$

Brasil-Neto JP, McShane LM, Fuhr P, Hallett M, Cohen LG (1992): Topographic 
mapping of the human motor cortex with magnetic stimulation: factors affecting accuracy and reproducibility. Electroencephalogr Clin Neurophysiol $\underline{85}, 9-16$

Cocito D, Cassano D (1993): Magnetic evoked responses elicited in the frontalis muscle. J Neurol Neurosurg Psychiatry $\underline{56}, 226$

Cody DT, Bickford RG (1969): Averaged evoked myogenic responses in normal man. Laryngoscope $\underline{79}, 400-16$

Cruccu G, Berardelli A, Inghilleri M, Manfredi M (1990): Corticobulbar projections to upper and lower facial motoneurons. A study by magnetic transcranial stimulation in man. Neurosci Lett $\underline{117}, 68-73$

Cruccu G, Inghilleri M, Berardelli A, Romaniello A, Manfredi M (1997): Cortical mechanisms mediating the inhibitory period after magnetic stimulation of the facial motor area. Muscle Nerve $\underline{20}, 418-24$

Darwin C: On the origin of species by means of natural selection, or the preservation of favoured races in the struggle for life. John Murray, London 1859

Day BL, Dressler D, Maertens de Noordhout A, Marsden CD, Nakashima K, Rothwell JC, Thompson PD (1989): Electric and magnetic stimulation of human motor cortex: surface EMG and single motor unit responses. J Physiol $\underline{412}$, 449-73

Denslow S, Bohning DE, Bohning PA, Lomarev MP, George MS (2005): An increased precision comparison of TMS-induced motor cortex BOLD fMRI response for image-guided versus function-guided coil placement. Cogn Behav Neurol 18, 119-26

Di Lazzaro V, Oliviero A, Profice P, Saturno E, Pilato F, Insola A, Mazzone P, Tonali P, Rothwell JC (1998): Comparison of descending volleys evoked by transcranial magnetic and electric stimulation in conscious humans. Electroen- 
cephalogr Clin Neurophysiol 109, 397-401

Dubach P, Guggisberg AG, Rosler KM, Hess CW, Mathis J (2004): Significance of coil orientation for motor evoked potentials from nasalis muscle elicited by transcranial magnetic stimulation. Clin Neurophysiol $\underline{115}, 862-70$

Ellaway PH, Davey NJ, Maskill DW, Rawlinson SR, Lewis HS, Anissimova NP (1998): Variability in the amplitude of skeletal muscle responses to magnetic stimulation of the motor cortex in man. Electroencephalogr Clin Neurophysiol $\underline{109}, 104-13$

English AW, Weeks OI (1989): Electromyographic cross-talk within a compartmentalized muscle of the cat. J Physiol $\underline{416}, 327-36$

Ettinger GJ, Leventon ME, Grimson WE, Kikinis R, Gugino L, Cote W, Sprung L, Aglio L, Shenton ME, Potts G, Hernandez VL, Alexander E (1998): Experimentation with a transcranial magnetic stimulation system for functional brain mapping. Med Image Anal 2, 133-42

Faraday M (1832): Experimental research in electricity. Philosophical Transactions $\underline{122}, 125-162$

Fischer U, Hess CW, Rosler KM (2005): Uncrossed cortico-muscular projections in humans are abundant to facial muscles of the upper and lower face, but may differ between sexes. J Neurol 252, 21-6

Fox PT, Narayana S, Tandon N, Sandoval H, Fox SP, Kochunov P, Lancaster JL (2004): Column-based model of electric field excitation of cerebral cortex. Hum Brain Mapp 22, 1-14

George MS, Lisanby SH, Avery D, McDonald WM, Durkalski V, Pavlicova M, Anderson B, Nahas Z, Bulow P, Zarkowski P, Holtzheimer PE, 3rd, Schwartz T, Sackeim HA (2010): Daily left prefrontal transcranial magnetic stimulation therapy for major depressive disorder: a sham-controlled randomized trial. 
Arch Gen Psychiatry $\underline{67}, 507-16$

Ghezzi A, Callea L, Zaffaroni M, Zibetti A (1992): Motor potentials of inferior orbicularis oculi muscle to transcranial magnetic stimulation. Comparison with responses to electrical peripheral stimulation of facial nerve. Electroencephalogr Clin Neurophysiol $\underline{85}, 248-52$

Guerra A, Petrichella S, Vollero L, Ponzo D, Pasqualetti P, Maatta S, Mervaala E, Kononen M, Bressi F, lannello G, Rossini PM, Ferreri F (2014): Neurophysiological features of motor cortex excitability and plasticity in Subcortical Ischemic Vascular Dementia: A TMS mapping study. Clin Neurophysiol Guggisberg AG, Dubach P, Hess CW, Wuthrich C, Mathis J (2001): Motor evoked potentials from masseter muscle induced by transcranial magnetic stimulation of the pyramidal tract: the importance of coil orientation. Clin Neurophysiol $\underline{112}, 2312-9$

Gugino LD, Romero JR, Aglio L, Titone D, Ramirez M, Pascual-Leone A, Grimson E, Weisenfeld N, Kikinis R, Shenton ME (2001): Transcranial magnetic stimulation coregistered with MRI: a comparison of a guided versus blind stimulation technique and its effect on evoked compound muscle action potentials. Clin Neurophysiol $\underline{112}, 1781-92$

Hackley SA (1993): An evaluation of the automaticity of sensory processing using event-related potentials and brain-stem reflexes. Psychophysiology $\underline{30}$, $415-28$

Henle J: Handbuch der Muskellehre des Menschen. In: Handbuch der systematischen Anatomie des Menschen. Bd. 1, Abt. 3; Vieweg, Braunschweig 1858

Henle J: Handbuch der Nervenlehre des Menschen. In: Handbuch der systematischen Anatomie des Menschen. Bd. 3, Abt. 2; Vieweg, Braunschweig 
1871

Herwig U, Kolbel K, Wunderlich AP, Thielscher A, von Tiesenhausen C, Spitzer M, Schonfeldt-Lecuona C (2002): Spatial congruence of neuronavigated transcranial magnetic stimulation and functional neuroimaging. Clin Neurophysiol $\underline{113}, 462-8$

Herwig U, Satrapi P, Schonfeldt-Lecuona C (2003): Using the international 1020 EEG system for positioning of transcranial magnetic stimulation. Brain Topogr $\underline{16}, 95-9$

Hess CW, Ludin HP (1988): [Transcranial cortex stimulation with magnetic field pulses: methodologic and physiologic principles]. EEG EMG Z Elektroenzephalogr Elektromyogr Verwandte Geb 19, 209-15

Hess CW, Mills KR, Murray NMF (1986): Magnetic stimulation of the human brain:

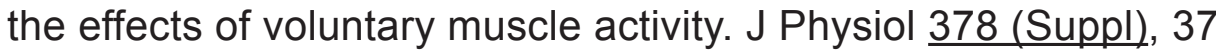

Heuser M (1976): [The oculoauricular phenomenon: a new reflex, a new muscle -- m. retroauricularis (polygraphic EMG study) (author's transl)]. J Neurol $\underline{212}, 95-9$

Hewitt M (2011-2012): Unveröffentliches Signal-Softwareskript (CED Signal v4.08) Jasper HH (1958): The ten-twenty electrode system of the International Federation. Electroencephalogr Clin Neurophysiol 10, 371-375

Jenny AB, Saper CB (1987): Organization of the facial nucleus and corticofacial projection in the monkey: a reconsideration of the upper motor neuron facial palsy. Neurology $\underline{37}, 930-9$

Julkunen P, Saisanen L, Danner N, Niskanen E, Hukkanen T, Mervaala E, Kononen M (2009): Comparison of navigated and non-navigated transcranial magnetic stimulation for motor cortex mapping, motor threshold and motor evoked potentials. Neuroimage $\underline{44}, 790-5$ 
Jung NH, Delvendahl I, Kuhnke NG, Hauschke D, Stolle S, Mall V (2010): Navigated transcranial magnetic stimulation does not decrease the variability of motor-evoked potentials. Brain Stimul $\underline{3}, 87-94$

Kantelhardt SR, Fadini T, Finke M, Kallenberg K, Siemerkus J, Bockermann V, Matthaeus L, Paulus W, Schweikard A, Rohde V, Giese A (2010): Robotassisted image-guided transcranial magnetic stimulation for somatotopic mapping of the motor cortex: a clinical pilot study. Acta Neurochir (Wien) $152,333-43$

Kiang NY-S, Crist, A H, French M A, Edwards A G (1963): Postauricular electric response to acoustic stimuli in humans. Quarterly Progress Report, Massachusetts Institute of Technology.

Kiers L, Cros D, Chiappa KH, Fang J (1993): Variability of motor potentials evoked by transcranial magnetic stimulation. Electroencephalogr Clin Neurophysiol $\underline{89}, 415-23$

Kiziltan ME, Gunduz A, Sahin R (2010): Auditory evoked blink reflex and posterior auricular muscle response: observations in patients with HFS and PFS. J Electromyogr Kinesiol 20, 508-12

Kleim JA, Kleim ED, Cramer SC (2007): Systematic assessment of training-induced changes in corticospinal output to hand using frameless stereotaxic transcranial magnetic stimulation. Nat Protoc 2, $1675-84$

Kobayashi M, Pascual-Leone A (2003): Transcranial magnetic stimulation in neurology. Lancet Neurol 2003, II, 145-56

Kobayashi M, Theoret H, Mottaghy FM, Gangitano M, Pascual-Leone A (2001): Intracortical inhibition and facilitation in human facial motor area: difference between upper and lower facial area. Clin Neurophysiol 112, 1604-11

Krings T, Buchbinder BR, Butler WE, Chiappa KH, Jiang HJ, Cosgrove GR, Rosen 
BR (1997): Functional magnetic resonance imaging and transcranial magnetic stimulation: complementary approaches in the evaluation of cortical motor function. Neurology $\underline{48}, 1406-16$

Krings T, Naujokat C, von Keyserlingk DG (1998): Representation of cortical motor function as revealed by stereotactic transcranial magnetic stimulation. Electroencephalogr Clin Neurophysiol 109, 85-93

Laakso I, Hirata A, Ugawa Y (2014): Effects of coil orientation on the electric field induced by TMS over the hand motor area. Phys Med Biol $\underline{59}$, 203-18

Lang N, Rothkegel H, Reiber H, Hasan A, Sueske E, Tergau F, Ehrenreich H, Wuttke W, Paulus W (2011): Circadian modulation of GABA-mediated cortical inhibition. Cereb Cortex 21, 2299-306

Littmann AE, McHenry CL, Shields RK (2013): Variability of motor cortical excitability using a novel mapping procedure. J Neurosci Methods $\underline{214}$, 137-43

Lotze M, Erb M, Flor H, Huelsmann E, Godde B, Grodd W (2000): fMRI evaluation of somatotopic representation in human primary motor cortex. Neuroimage $11,473-81$

Lotze M, Kaethner RJ, Erb M, Cohen LG, Grodd W, Topka H (2003): Comparison of representational maps using functional magnetic resonance imaging and transcranial magnetic stimulation. Clin Neurophysiol 114, 306-12

Maccabee PJ, Amassian VE, Eberle LP, Cracco RQ (1993): Magnetic coil stimulation of straight and bent amphibian and mammalian peripheral nerve in vitro: locus of excitation. J Physiol $\underline{460}, 201-19$

Malcolm MP, Triggs WJ, Light KE, Shechtman O, Khandekar G, Gonzalez Rothi LJ (2006): Reliability of motor cortex transcranial magnetic stimulation in four muscle representations. Clin Neurophysiol 117, 1037-46

Mangun GR, Mulkey RM, Young BL, Goslow GE, Jr. (1986): “Cross-talk” in elec- 
tromyograms: contamination of EMGs recorded with bipolar fine-wire electrodes by volume conducted myoelectric activity from distant sources. Electromyogr Clin Neurophysiol 26, 443-61

Mansur CG, Fregni F, Boggio PS, Riberto M, Gallucci-Neto J, Santos CM, Wagner T, Rigonatti SP, Marcolin MA, Pascual-Leone A (2005): A sham stimulationcontrolled trial of rTMS of the unaffected hemisphere in stroke patients. Neurology $\underline{64}, 1802-4$

McNamara B, Ray JL, Arthurs OJ, Boniface S (2001): Transcranial magnetic stimulation for depression and other psychiatric disorders. Psychol Med $\underline{31}$, $1141-6$

Meier JD, Aflalo TN, Kastner S, Graziano MS (2008): Complex organization of human primary motor cortex: a high-resolution fMRI study. J Neurophysiol $\underline{100}, 1800-12$

Merton PA, Morton HB (1980): Stimulation of the cerebral cortex in the intact human subject. Nature $\underline{285}, 227$

Mills KR, Boniface SJ, Schubert M (1992): Magnetic brain stimulation with a double coil: the importance of coil orientation. Electroencephalogr Clin Neurophysiol $\underline{85}, 17-21$

Morecraft RJ, Louie JL, Herrick JL, Stilwell-Morecraft KS (2001): Cortical innervation of the facial nucleus in the non-human primate: a new interpretation of the effects of stroke and related subtotal brain trauma on the muscles of facial expression. Brain 124, 176-208

O’Beirne GA, Patuzzi RB (1999): Basic properties of the sound-evoked post-auricular muscle response (PAMR). Hear Res $\underline{138}, 115-32$

Oldfield RC (1971): The assessment and analysis of handedness: the Edinburgh inventory. Neuropsychologia $\underline{9}, 97-113$ 
Orth M, Rothwell JC (2004): The cortical silent period: intrinsic variability and relation to the waveform of the transcranial magnetic stimulation pulse. Clin Neurophysiol $\underline{115}, 1076-82$

Paradiso GO, Cunic DI, Gunraj CA, Chen R (2005): Representation of facial muscles in human motor cortex. J Physiol $\underline{567}, 323-36$

Penfield W, Boldrey E (1937): Somatic motor and sensory representation in the cerebral cortex of man as studied by electrical stimulation. Brain $\underline{60}, 389-$ 443

Purdy SC, Agung KB, Hartley D, Patuzzi RB, O’Beirne GA (2005): The post-auricular muscle response: an objective electrophysiological method for evaluating hearing sensitivity. Int J Audiol $\underline{44}$, 625-30

Rao BS, Desiraju T, Raju TR (1993): Neuronal plasticity induced by self-stimulation rewarding experience in rats--a study on alteration in dendritic branching in pyramidal neurons of hippocampus and motor cortex. Brain Res $\underline{627}$, 216-24

Reilly KT, Mercier C (2008): Cortical topography of human first dorsal interroseus during individuated and nonindividuated grip tasks. Hum Brain Mapp $\underline{29}$, 594-602

Richter L, Neumann G, Oung S, Schweikard A, Trillenberg P (2013a): Optimal coil orientation for transcranial magnetic stimulation. PLoS One $\underline{8}$, e60358

Richter L, Trillenberg P, Schweikard A, Schlaefer A (2013b): Stimulus intensity for hand held and robotic transcranial magnetic stimulation. Brain Stimul $\underline{6}$, $315-21$

Ridding MC, Rothwell JC (2007): Is there a future for therapeutic use of transcranial magnetic stimulation? Nat Rev Neurosci $\underline{8}, 559-67$

Rödel R, Laskawi R, Markus H (2000): Motor potentials of lower-lip mimetic mus- 
cles and distal arm muscles to cortical transcranial magnetic stimulation: the possibility of one-dimensional separation of two cortical representation areas. ORL J Otorhinolaryngol Relat Spec 62, 96-9

Roick H, von Giesen HJ, Benecke R (1993): On the origin of the postexcitatory inhibition seen after transcranial magnetic brain stimulation in awake human subjects. Exp Brain Res $\underline{94}$, 489-98

Rossini PM, Barker AT, Berardelli A, Caramia MD, Caruso G, Cracco RQ, Dimitrijevic MR, Hallett M, Katayama Y, Lücking $\mathrm{CH}$, Maertens de Noordhout $\mathrm{AL}$, Marsden CD, Murray NMF, Rothwell JC, Swash M, Tomberg C (1994): Noninvasive electrical and magnetic stimulation of the brain, spinal cord and roots: basic principles and procedures for routine clinical application. Report of an IFCN committee. Electroencephalogr Clin Neurophysiol 91, 79-92

Rossini PM, Caramia MD, Zarola F (1987): Mechanisms of nervous propagation along central motor pathways: noninvasive evaluation in healthy subjects and in patients with neurological disease. Neurosurgery $\underline{20}, 183-91$

Rothwell JC, Hallett M, Berardelli A, Eisen A, Rossini P, Paulus W (1999): Magnetic stimulation: motor evoked potentials. The International Federation of Clinical Neurophysiology. Electroencephalogr Clin Neurophysiol Suppl $\underline{52}$, $97-103$

Rusu CV, Murakami M, Ziemann U, Triesch J (2014): A model of TMS-induced Iwaves in motor cortex. Brain Stimul $\underline{7}, 401-14$

Schmidt D, Thoden U (1978): Co-activation of the M. transversus auris with eye movements (Wilson's oculo-auricular phenomenon) and with activity in other cranial nerves. Albrecht Von Graefes Arch Klin Exp Ophthalmol 206, 227-36

Schwalbe G: Sinnesorgane. 1. Das äußere Ohr. In: Handbuch der Anatomie des Menschen. Bd 5, 2. Abt.; Gustav Fischer, Jena 1897 
Serra G, Tugnoli V, Cristofori MC, Eleopra R, De Grandis D (1986): The electromyographic examination of the posterior auricular muscle. Electromyogr Clin Neurophysiol 26, 661-5

Sohn YH, Voller B, Dimyan M, St Clair Gibson A, Hanakawa T, Leon-Sarmiento FE, Jung HY, Hallett M (2004): Cortical control of voluntary blinking: a transcranial magnetic stimulation study. Clin Neurophysiol $\underline{115}, 341-7$

Sparing R, Buelte D, Meister IG, Paus T, Fink GR (2008): Transcranial magnetic stimulation and the challenge of coil placement: a comparison of conventional and stereotaxic neuronavigational strategies. Hum Brain Mapp $\underline{29}$, $82-96$

Terao Y, Ugawa Y, Sakai K, Miyauchi S, Fukuda H, Sasaki Y, Takino R, Hanajima R, Furubayashi T, Putz B, Kanazawa I (1998): Localizing the site of magnetic brain stimulation by functional MRI. Exp Brain Res $\underline{121}, 145-52$

Urban PP, Marczynski U, Hopf HC (1993): The oculo-auricular phenomenon. Findings in normals and patients with brainstem lesions. Brain 116 (Pt 3), 72738

Wassermann EM, McShane LM, Hallett M, Cohen LG (1992): Noninvasive mapping of muscle representations in human motor cortex. Electroencephalogr Clin Neurophysiol $\underline{85}, 1-8$

Wassermann EM, Pascual-Leone A, Valls-Sole J, Toro C, Cohen LG, Hallett M (1993): Topography of the inhibitory and excitatory responses to transcranial magnetic stimulation in a hand muscle. Electroencephalogr Clin Neurophysiol $\underline{89}, 424-33$

Wassermann EM, Pascual-Leone A, Hallett M (1994): Cortical motor representation of the ipsilateral hand and arm. Exp Brain Res $\underline{100}, 121-32$

Wassermann EM (2002): Variation in the response to transcranial magnetic brain 
stimulation in the general population. Clin Neurophysiol $\underline{113}, 1165-71$

Weiss C, Nettekoven C, Rehme AK, Neuschmelting V, Eisenbeis A, Goldbrunner R, Grefkes C (2012): Mapping the hand, foot and face representations in the primary motor cortex - Retest reliability of neuronavigated TMS versus functional MRI. Neuroimage $\underline{66 \mathrm{C}}, 531-542$

Weyh T, Siebner HR: Hirnstimulation - Technische Grundlagen. In: Das TMS Buch, Handbuch der transkraniellen Magnetstimulation. Hrsg. v. Siebner HR, Ziemann U; Springer Medizin Verlag, Heidelberg 2007, 17-26

Wilson SA, Lockwood RJ, Thickbroom GW, Mastaglia FL (1993): The muscle silent period following transcranial magnetic cortical stimulation. J Neurol Sci $114,216-22$

Wilson SAK (1908): A note on an associated movement of the eyes and ears in man. Rev Neurol Psychiatry $\underline{6}, 331-336$

Ziemann U, Rothwell JC, Ridding MC (1996): Interaction between intracortical inhibition and facilitation in human motor cortex. J Physiol 496 ( Pt 3), 873-81 


\section{Lebenslauf}

Mein Name ist Jonna Meincke. Ich bin ledig und wohne im Kreuzbergring 4a in 37075 Göttingen. Geboren wurde ich am 5. Januar 1987 in Göttingen.

Nach einem einjährigen Auslandsaufenthalt in Chile während der Schulzeit kehrte ich nach meinem Abitur 2006 (Note: 1,1) für einen einjährigen Freiwilligendienst nach Chile zurück. Durch beide Auslandsaufenthalte motiviert und mit dem Wunsch nach einem menschennahen und von Staatsgrenzen unabhängigen Beruf, begann ich 2007 mein Medizinstudium an der Georg-August-Universität in Göttingen, das ich im November 2013 mit der Note 1,7 abschloss.

Schon während des vorklinischen Studienabschnitts weckte besonders das Themengebiet Neuroanatomie und -physiologie mein Interesse, das sich durch die Belegung zahlreicher Wahlfächer und die Absolvierung diverser Famulaturen im klinischen Teil des Medizinstudiums intensivierte. Vor diesem Hintergrund begann ich im Sommer 2010 als studentische Hilfskraft in der Klinik für Klinische Neurophysiologie in der Arbeitsgruppe von Prof. Liebetanz mit einer experimentellen Forschungstätigkeit. Hier wirkte ich in der Etablierung einer robotergestützten und neuronavigationsbasierten transkraniellen Magnetstimulation sowie ihrer Anwendung mit und hatte die Gelegenheit, meine Ergebnisse 2012 auf dem Meeting der American Society for Neuroscience in New Orleans vorzustellen. Die vielfältigen Forschungsbereiche, in die ich dabei Einblick gewinnen konnte, sowie der in der erwähnten Arbeitsgruppe gewährte Freiraum für eigene Ideen und Konzepte motivierte mich zu weiterführender experimenteller Forschung, sodass ich nach meiner Approbation als Ärztin begann, in der Klinik für Klinische Neurophysiologie als 
wissenschaftliche Mitarbeiterin zu arbeiten. Im Rahmen dieser Tätigkeit möchte ich an meine bisherige Forschungsarbeit anknüpfen und mein wissenschaftliches Engagement unter Anwendung der robotergestützten und neuronavigationsbasierten transkraniellen Magnetstimulation weiter intensivieren. 


\section{Danksagung}

Ich bedanke mich ganz herzlich bei David Liebetanz für die Bereitstellung des interessanten Themas, das Vertrauen dieses Projekt zu betreuen und ganz besonders für die Freiheit meine eigenen Ideen zu entwickeln und einzubringen. Ich danke für die intensive Betreuung während der gesamten Projektdauer, die Möglichkeit das wissenschaftliche Arbeiten zu erlernen und frei zu experimentieren. Auch bedanke ich mich für die zahlreichen Gespräche und Anregungen und das große Maß an Geduld insbesondere während der Beeindigung des Projektes. Durch die Zusammenarbeit mit David Liebetanz entstand mein bis heute währendes Interesse an der Wissenschaft.

Mein ganz besonderer Dank gilt Manuel Hewitt, meinem wissenschaftlichen Begleiter, Freund und Diskussionspartner. Ohne seine Voruntersuchungen am Roboter und seinen akribischen Perfektionismus hätte das Projekt in der Form nicht stattfinden können. Ich danke für die interessanten Anregungen, die Chance unterschiedlichsten Ideen nachzugehen und auszutesten und für die Hartnäckigkeit während der Bewältigung von unlösbar erscheinenden Aufgaben. Neben dem Vorantreiben des Projektes habe ich die vielen konspirativen Stunden im Labor und die zahlreichen Gespräche als eine große Bereicherung empfunden, die meinen Lebensweg, sowohl professionell als auch persönlich, in großem Maße beeinflusst haben.

Mein Dank geht ebenso an die Kollegen der Klinik für Klinische Neurophysiologie, die mir jederzeit für Fragen zur Verfügung standen und mich herzlich in ihr Team aufgenommen haben. 
Ich bedanke mich bei allen Probanden, die an der Studie oder den zahlreichen Voruntersuchungen teilgenommen haben und ohne die das Projekt nicht funktioniert hätte. Ich danke insbesondere den ersten Probanden für ihren Mut und die Geduld beim experimentieren.

Abschließend möchte ich mich bei meiner Mutter und Schwester für die Ideen und Anregungen beim Anfertigen dieser Arbeit bedanken. 

